\title{
FERMI LARGE AREA TELESCOPE OBSERVATIONS OF MARKARIAN 421: THE MISSING PIECE OF ITS SPECTRAL ENERGY DISTRIBUTION
}

A. A. Abdo ${ }^{1,110}$, M. AckermanN ${ }^{2}$, M. Ajello ${ }^{2}$, L. Baldini ${ }^{3}$, J. Ballet ${ }^{4}$, G. Barbiellini ${ }^{5,6}$, D. Bastieri ${ }^{7,8}$, K. BeChtol $^{2}$, R. Bellazzini ${ }^{3}$, B. Berenji ${ }^{2}$, R. D. Blandford ${ }^{2}$, E. D. Bloom ${ }^{2}$, E. Bonamente ${ }^{9,10}$, A. W. Borgland ${ }^{2}$, A. BouvieR ${ }^{11}$, J. Bregeon ${ }^{3}$, A. Brez ${ }^{3}$, M. Brigida ${ }^{12,13}$, P. Bruel ${ }^{14}$, R. Buehler ${ }^{2}$, S. Buson ${ }^{7,8}$, G. A. Caliandro ${ }^{15}$, R. A. Cameron ${ }^{2}$, A. Cannon ${ }^{16,17}$, P. A. Caraveo ${ }^{18}$, S. Carrigan ${ }^{8}$, J. M. Casandjian ${ }^{4}$, E. Cavazzuti ${ }^{19}$, C. Cecchi ${ }^{9}, 10$, Ö. Çelik ${ }^{16,20,21}$, E. Charles ${ }^{2}$, A. Chekhtman ${ }^{22,110}$, J. Chiang ${ }^{2}$, S. Ciprini ${ }^{10}$, R. Clauus ${ }^{2}$, J. Cohen-Tanugi ${ }^{23}$, J. Conrad ${ }^{24,25,111}$, S. Cutini ${ }^{19}$, A. De Angelis ${ }^{26}$, F. De Palma ${ }^{12,13}$, C. D. Dermer ${ }^{27}$, E. do Couto e Silva ${ }^{2}$, P. S. Drell ${ }^{2}$, R. Dubois ${ }^{2}$, D. Dumora ${ }^{28}$, L. Escande ${ }^{28,29}$, C. FAvuZzi ${ }^{12,13}$, S. J. Fegan ${ }^{14}$, J. FinKe ${ }^{27}$, W. B. FocKe ${ }^{2}$, P. Fortin ${ }^{14}$, M. Frailis ${ }^{26,30}$, L. FuhrmanN ${ }^{31}$, Y. Fukazawa ${ }^{32}$, T. Fukuyama ${ }^{33}$, S. FunK ${ }^{2}$, P. Fusco ${ }^{12,13}$, F. Gargano ${ }^{13}$, D. Gasparrini ${ }^{19}$, N. Gehrels ${ }^{16}$,

M. Georganopoulos ${ }^{21}$, S. Germani ${ }^{9}, 10$, B. Giebels ${ }^{14}$, N. Giglietto ${ }^{12,13}$, P. Giommi ${ }^{19}$, F. Giordano ${ }^{12,13}$, M. Giroletti ${ }^{34}$,

T. Glanzman ${ }^{2}$, G. Godfrey ${ }^{2}$, I. A. Grenier ${ }^{4}$, S. Guiriec ${ }^{35}$, D. Hadasch ${ }^{15}$, M. Hayashida ${ }^{2}$, E. Hays ${ }^{16}$, D. Horan ${ }^{14}$,

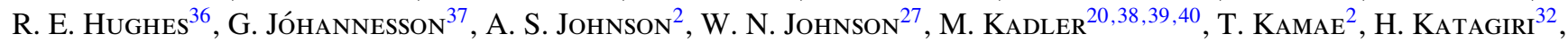

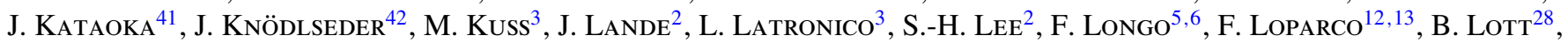
M. N. Lovellette ${ }^{27}$, P. Lubrano ${ }^{9,10}$, G. M. Madejski ${ }^{2}$, A. Makeev ${ }^{22,110}$, W. Max-Moerbeck ${ }^{43}$, M. N. Mazziotta ${ }^{13}$, J. E. McEneri ${ }^{16,44}$, J. Mehault ${ }^{23}$, P. F. Michelson ${ }^{2}$, W. Mitthumsiri ${ }^{2}$, T. Mizuno ${ }^{32}$, C. Monte ${ }^{12,13}$, M. E. Monzani ${ }^{2}$,

A. Morsellit ${ }^{45}$, I. V. Moskalenko ${ }^{2}$, S. Murgia ${ }^{2}$, T. Nakamori ${ }^{41}$, M. Naumann-Godo ${ }^{4}$, S. Nishino ${ }^{32}$, P. L. Nolan ${ }^{2}$, J. P. Norris ${ }^{46}$, E. Nuss ${ }^{23}$, T. Ohsugi ${ }^{47}$, A. OkUmura ${ }^{33}$, N. OmodeI ${ }^{2}$, E. Orlando ${ }^{2,48}$, J. F. Ormes ${ }^{46}$, M. Ozaki ${ }^{33}$,

D. Paneque ${ }^{2,49}$, J. H. Panetta ${ }^{2}$, D. Parent ${ }^{22,110}$, V. Pavlidou ${ }^{43}$, T. J. Pearson ${ }^{43}$, V. Pelassa ${ }^{23}$, M. Pepe Pe, $^{9}$,

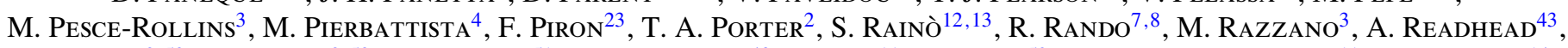
A. Reimer ${ }^{2,50}$, O. Reimer ${ }^{2,50}$, L. C. ReYes ${ }^{51}$, J. L. Richards ${ }^{43}$, S. Ritz ${ }^{11}$, M. Roth ${ }^{52}$, H. F.-W. SAdrozinski ${ }^{11}$, D. SANCHeZ ${ }^{14}$,

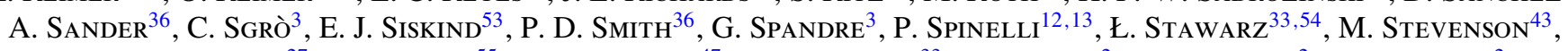
M. S. Strickman ${ }^{27}$, D. J. SusON ${ }^{55}$, H. TAKAhashi ${ }^{47}$, T. TAKAhashi ${ }^{33}$, T. TANAKA ${ }^{2}$, J. G. ThaYer ${ }^{2}$, J. B. Thayer ${ }^{2}$, D. J. Thompson ${ }^{16}$, L. Tibaldo ${ }^{4,7,8,112}$, D. F. Torres ${ }^{15,56}$, G. Tosti $^{9,10}$, A. Tramacere ${ }^{2,57,58}$, E. Troja ${ }^{16,113}$, T. L. Usher ${ }^{2}$, J. Vandenbroucke ${ }^{2}$, V. Vasileiou ${ }^{20,21}$, G. Vianello ${ }^{2,57}$, N. Vilchez ${ }^{42}$, V. Vitale ${ }^{45,59}$, A. P. Waite ${ }^{2}$, P. Wang $^{2}$, A. E. WeHrLE ${ }^{60}$, B. L. WINER ${ }^{36}$, K. S. WOOD ${ }^{27}$, Z. YANG ${ }^{24,25}$, Y. YATSU ${ }^{61}$, T. YLINEN ${ }^{25,62,63}$, J. A. Zensus ${ }^{31}$, M. ZIEGLER ${ }^{11}$

(The Fermi-LAT Collaboration)

J. Aleksić ${ }^{64}$, L. A. Antonelli ${ }^{65}$, P. Antoranz ${ }^{66}$, M. Backes ${ }^{67}$, J. A. Barrio ${ }^{68}$, J. Becerra González ${ }^{69,70}$, W. BednareK ${ }^{71}$, A. Berdyugin ${ }^{72}$, K. Berger ${ }^{70}$, E. Bernardini ${ }^{73}$, A. Biland ${ }^{74}$, O. Blanch ${ }^{64}$, R. K. Bock ${ }^{49}$, A. Boller ${ }^{74}$, G. BonNOLI ${ }^{65}$, P. Bordas ${ }^{75}$, D. Borla Tridon ${ }^{49}$, V. Bosch-Ramon ${ }^{75}$, D. Bose ${ }^{68}$, I. Braun ${ }^{74}$, T. Bretz ${ }^{76}$, M. Camara ${ }^{68}$, E. CARmona ${ }^{49}$, A. Carosi ${ }^{65}$, P. Colin ${ }^{49}$, E. Colombo ${ }^{69}$, J. L. Contreras ${ }^{68}$, J. Cortina ${ }^{64}$, S. Covino ${ }^{65}$, F. Dazzi ${ }^{26,114}$, A. De Angelis ${ }^{26}$, E. De Cea del Pozo ${ }^{15}$, C. Delgado Mendez ${ }^{69,77}$, B. De Lotto $^{78}$, M. De Maria ${ }^{78}$, F. De Sabata ${ }^{78}$, A. Diago Ortega ${ }^{69,70}$,

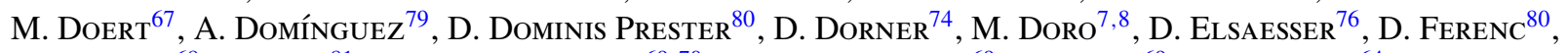
M. V. FonseCa ${ }^{68}$, L. FonT ${ }^{81}$, R. J. García LóPez ${ }^{69,70}$, M. GarCZarCZYK ${ }^{69}$, M. Gaug ${ }^{69}$, G. Giavitto ${ }^{64}$, N. GodinOvi ${ }^{80}$,

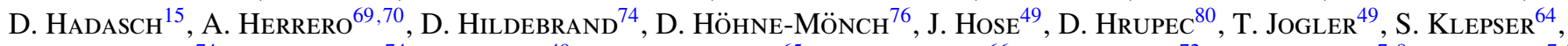

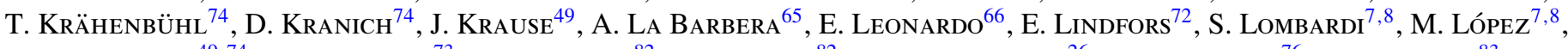

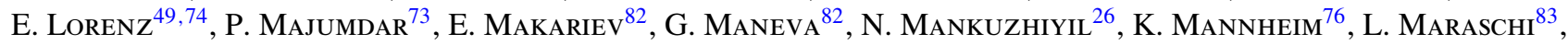
M. Mariottit ${ }^{7}, 8$, M. Martínez ${ }^{64}$, D. Mazin ${ }^{64}$, M. Meucci $^{66}$, J. M. Miranda ${ }^{66}$, R. Mirzoyan ${ }^{49}$, H. Miyamoto ${ }^{49}$, J. Moldón ${ }^{75}$, A. Moralejo ${ }^{64}$, D. Nieto ${ }^{68}$, K. Nilsson ${ }^{84}$, R. Orito ${ }^{49}$, I. Oya ${ }^{68}$, R. PaOletti ${ }^{66}$, J. M. Paredes ${ }^{75}$, S. Partini ${ }^{66}$, M. Pasanen ${ }^{72}$, F. Pauss ${ }^{74}$, R. G. Pegna ${ }^{66}$, M. A. Perez-Torres ${ }^{79}$, M. Persic ${ }^{26,85}$, J. Peruzzo ${ }^{7,8}$, J. Pochon ${ }^{69}$, F. Prada ${ }^{79}$,

P. G. Prada Moroni ${ }^{66}$, E. Prandini ${ }^{7,8}$, N. Puchades ${ }^{64}$, I. Puljak ${ }^{80}$, T. Reichardt $^{64}$, W. Rhode ${ }^{67}$, M. Ribó ${ }^{75}$, J. Rico ${ }^{56,64}$,

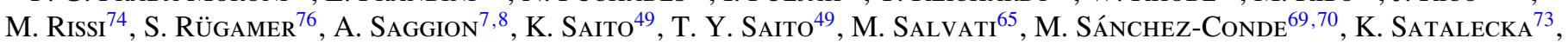
V. Scalzotto ${ }^{7,8}$, V. Scapin ${ }^{26}$, C. Schultz ${ }^{7,8}$, T. Schweizer ${ }^{49}$, M. ShaYduK ${ }^{49}$, S. N. Shore ${ }^{3,86}$, A. Sierpowska-Bartosik ${ }^{71}$, A. Sillanpää ${ }^{72}$, J. Sitarek ${ }^{49,71}$, D. Sobczynska ${ }^{71}$, F. Spanier ${ }^{76}$, S. Spiro ${ }^{65}$, A. Stamerra ${ }^{66}$, B. Steinke ${ }^{49}$, J. Storz ${ }^{76}$,

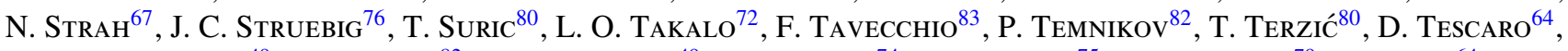

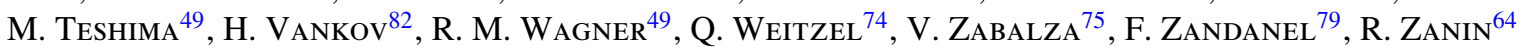
(The MAGiC Collaboration)

AND

M. Villata ${ }^{87}$, C. Raiteri ${ }^{87}$, H. D. Aller ${ }^{88}$, M. F. Aller ${ }^{88}$, W. P. Chen ${ }^{89}$, B. Jordan ${ }^{90}$, E. Koptelova ${ }^{89}$, O. M. Kurtanidze ${ }^{91}$, A. LähteenmäKi ${ }^{92}$, B. McBreen ${ }^{17}$, V. M. Larionov ${ }^{93,94,95}$, C. S. Lin ${ }^{89}$, M. G. NikolashVili ${ }^{91}$, R. Reinthal ${ }^{72}$, E. Angelakis ${ }^{31}$, M. Capalbi ${ }^{19}$, A. Carramiñana ${ }^{96}$, L. Carrasco $^{96}$, P. Cassaro ${ }^{97}$, A. Cesarini ${ }^{98}$, A. Falcone ${ }^{99}$, M. A. Gurwell ${ }^{100}$, T. Hovatta ${ }^{92}$, Yu. A. Kovalev ${ }^{101}$, Y. Y. Kovalev ${ }^{31,101}$, T. P. Krichbaum ${ }^{31}$, H. A. Krimm ${ }^{20,40}$, M. L. Lister ${ }^{102}$, J. W. Moody ${ }^{103}$, G. MacCAFerri ${ }^{104}$, Y. Mori $^{61}$, I. Nestoras ${ }^{31}$, A. Orlati ${ }^{104}$, C. Pace ${ }^{103}$, C. Pagani ${ }^{105}$, R. Pearson ${ }^{103}$, M. Perri ${ }^{19}$, B. G. Piner ${ }^{106}$, E. Ros $^{31,107}$, A. C. SAdun ${ }^{108}$, T. SAKAMOTO ${ }^{16}$, J. TAMmi ${ }^{92}$, AND A. ZoOK ${ }^{109}$ 
${ }^{1}$ National Research Council Research Associate, National Academy of Sciences, Washington, DC 20001, USA

${ }^{2}$ W. W. Hansen Experimental Physics Laboratory, Kavli Institute for Particle Astrophysics and Cosmology, Department of Physics and SLAC National Accelerator Laboratory, Stanford University, Stanford, CA 94305, USA; dpaneque@mppmu.mpg.de, anita.reimer@uibk.ac.at

${ }^{3}$ Istituto Nazionale di Fisica Nucleare, Sezione di Pisa, I-56127 Pisa, Italy

${ }^{4}$ Laboratoire AIM, CEA-IRFU/CNRS/Université Paris Diderot, Service d'Astrophysique, CEA Saclay, 91191 Gif sur Yvette, France

${ }^{5}$ Istituto Nazionale di Fisica Nucleare, Sezione di Trieste, I-34127 Trieste, Italy

${ }^{6}$ Dipartimento di Fisica, Università di Trieste, I-34127 Trieste, Italy

${ }^{7}$ Istituto Nazionale di Fisica Nucleare, Sezione di Padova, I-35131 Padova, Italy

${ }^{8}$ Dipartimento di Fisica "G. Galilei," Università di Padova, I-35131 Padova, Italy

9 Istituto Nazionale di Fisica Nucleare, Sezione di Perugia, I-06123 Perugia, Italy

${ }^{10}$ Dipartimento di Fisica, Università degli Studi di Perugia, I-06123 Perugia, Italy

${ }^{11}$ Santa Cruz Institute for Particle Physics, Department of Physics and Department of Astronomy and Astrophysics, University of California at Santa Cruz, Santa Cruz, CA 95064, USA

12 Dipartimento di Fisica “M. Merlin" dell'Università e del Politecnico di Bari, I-70126 Bari, Italy

${ }^{13}$ Istituto Nazionale di Fisica Nucleare, Sezione di Bari, 70126 Bari, Italy

${ }^{14}$ Laboratoire Leprince-Ringuet, École polytechnique, CNRS/IN2P3, Palaiseau, France

${ }^{15}$ Institut de Ciencies de l'Espai (IEEC-CSIC), Campus UAB, 08193 Barcelona, Spain

${ }^{16}$ NASA Goddard Space Flight Center, Greenbelt, MD 20771, USA

${ }^{17}$ University College Dublin, Belfield, Dublin 4, Ireland

${ }^{18}$ INAF-Istituto di Astrofisica Spaziale e Fisica Cosmica, I-20133 Milano, Italy

${ }^{19}$ Agenzia Spaziale Italiana (ASI) Science Data Center, I-00044 Frascati (Roma), Italy

${ }^{20}$ Center for Research and Exploration in Space Science and Technology (CRESST) and NASA Goddard Space Flight Center, Greenbelt, MD 20771, USA

${ }^{21}$ Department of Physics and Center for Space Sciences and Technology, University of Maryland Baltimore County, Baltimore, MD 21250, USA; georgano@umbc.edu

${ }^{22}$ College of Science, George Mason University, Fairfax, VA 22030, USA

${ }^{23}$ Laboratoire de Physique Théorique et Astroparticules, Université Montpellier 2, CNRS/IN2P3, Montpellier, France

${ }^{24}$ Department of Physics, Stockholm University, AlbaNova, SE-106 91 Stockholm, Sweden

25 The Oskar Klein Centre for Cosmoparticle Physics, AlbaNova, SE-106 91 Stockholm, Sweden

${ }^{26}$ Dipartimento di Fisica, Università di Udine and Istituto Nazionale di Fisica Nucleare, Sezione di Trieste, Gruppo Collegato di Udine, I-33100 Udine, Italy

${ }^{27}$ Space Science Division, Naval Research Laboratory, Washington, DC 20375, USA; justin.finke@nrl.navy.mil

${ }^{28}$ Université Bordeaux 1, CNRS/IN2p3, Centre d'Études Nucléaires de Bordeaux Gradignan, 33175 Gradignan, France

${ }^{29}$ CNRS/IN2P3, Centre d'Études Nucléaires Bordeaux Gradignan, UMR 5797, Gradignan, 33175, France

${ }^{30}$ Osservatorio Astronomico di Trieste, Istituto Nazionale di Astrofisica, I-34143 Trieste, Italy

${ }^{31}$ Max-Planck-Institut für Radioastronomie, Auf dem Hügel 69, 53121 Bonn, Germany

${ }^{32}$ Department of Physical Sciences, Hiroshima University, Higashi-Hiroshima, Hiroshima 739-8526, Japan

${ }^{33}$ Institute of Space and Astronautical Science, JAXA, 3-1-1 Yoshinodai, Chuo-ku, Sagamihara, Kanagawa 252-5210, Japan

${ }^{34}$ INAF Istituto di Radioastronomia, 40129 Bologna, Italy

${ }^{35}$ Center for Space Plasma and Aeronomic Research (CSPAR), University of Alabama in Huntsville, Huntsville, AL 35899, USA

${ }^{36}$ Department of Physics, Center for Cosmology and Astro-Particle Physics, The Ohio State University, Columbus, OH 43210, USA

${ }^{37}$ Science Institute, University of Iceland, IS-107 Reykjavik, Iceland

${ }^{38}$ Dr. Remeis-Sternwarte Bamberg, Sternwartstrasse 7, D-96049 Bamberg, Germany

${ }^{39}$ Erlangen Centre for Astroparticle Physics, D-91058 Erlangen, Germany

${ }^{40}$ Universities Space Research Association (USRA), Columbia, MD 21044, USA

${ }^{41}$ Research Institute for Science and Engineering, Waseda University, 3-4-1, Okubo, Shinjuku, Tokyo, 169-8555 Japan

${ }^{42}$ Centre d'Étude Spatiale des Rayonnements, CNRS/UPS, BP 44346, F-30128 Toulouse Cedex 4, France

${ }^{43}$ Cahill Center for Astronomy and Astrophysics, California Institute of Technology, Pasadena, CA 91125, USA

${ }^{44}$ Department of Physics and Department of Astronomy, University of Maryland, College Park, MD 20742, USA

45 Istituto Nazionale di Fisica Nucleare, Sezione di Roma "Tor Vergata," I-00133 Roma, Italy

${ }^{46}$ Department of Physics and Astronomy, University of Denver, Denver, CO 80208, USA

${ }^{47}$ Hiroshima Astrophysical Science Center, Hiroshima University, Higashi-Hiroshima, Hiroshima 739-8526, Japan

${ }^{48}$ Max-Planck Institut für extraterrestrische Physik, 85748 Garching, Germany

${ }^{49}$ Max-Planck-Institut für Physik, D-80805 München, Germany

${ }^{50}$ Institut für Astro- und Teilchenphysik and Institut für Theoretische Physik, Leopold-Franzens-Universität Innsbruck, A-6020 Innsbruck, Austria

${ }^{51}$ Kavli Institute for Cosmological Physics, University of Chicago, Chicago, IL 60637, USA

52 Department of Physics, University of Washington, Seattle, WA 98195-1560, USA

${ }^{53}$ NYCB Real-Time Computing Inc., Lattingtown, NY 11560-1025, USA

${ }^{54}$ Astronomical Observatory, Jagiellonian University, 30-244 Kraków, Poland

55 Department of Chemistry and Physics, Purdue University Calumet, Hammond, IN 46323-2094, USA

${ }^{56}$ Institució Catalana de Recerca i Estudis Avançats (ICREA), Barcelona, Spain

${ }^{57}$ Consorzio Interuniversitario per la Fisica Spaziale (CIFS), I-10133 Torino, Italy

${ }^{58}$ INTEGRAL Science Data Centre, CH-1290 Versoix, Switzerland

${ }^{59}$ Dipartimento di Fisica, Università di Roma “Tor Vergata," I-00133 Roma, Italy

${ }^{60}$ Space Science Institute, Boulder, CO 80301, USA

${ }^{61}$ Department of Physics, Tokyo Institute of Technology, Meguro City, Tokyo 152-8551, Japan

62 Department of Physics, Royal Institute of Technology (KTH), AlbaNova, SE-106 91 Stockholm, Sweden

${ }^{63}$ School of Pure and Applied Natural Sciences, University of Kalmar, SE-391 82 Kalmar, Sweden

${ }^{64}$ Institut de Física d'Altes Energies (IFAE), Edifici Cn, Universitat Autònoma de Barcelona (UAB), E-08193 Bellaterra (Barcelona), Spain; diegot@ifae.es

65 INAF National Institute for Astrophysics, I-00136 Roma, Italy

${ }^{66}$ Università di Siena and INFN Pisa, I-53100 Siena, Italy

${ }^{67}$ Technische Universität Dortmund, D-44221 Dortmund, Germany

${ }^{68}$ Universidad Complutense, E-28040 Madrid, Spain

${ }^{69}$ Instituto de Astrofísica de Canarias, E38205 - La Laguna (Tenerife), Spain

${ }^{70}$ Departamento de Astrofisica, Universidad de La Laguna, E-38205 La Laguna, Tenerife, Spain 


\author{
${ }^{71}$ University of Łódź, PL-90236 Łódź, Poland \\ 72 Tuorla Observatory, University of Turku, FI-21500 Piikkiö, Finland \\ ${ }^{73}$ Deutsches Elektronen Synchrotron DESY, D-15738 Zeuthen, Germany \\ ${ }^{74}$ ETH Zurich, CH-8093 Zurich, Switzerland \\ ${ }^{75}$ Universitat de Barcelona (ICC/IEEC), E-08028 Barcelona, Spain \\ 76 Institut für Theoretische Physik and Astrophysik, Universität Würzburg, D-97074 Würzburg, Germany \\ ${ }^{77}$ Centro de Investigaciones Energéticas, Medioambientales y Tecnológicas (CIEMAT), Madrid, Spain \\ ${ }^{78}$ Istituto Nazionale di Fisica Nucleare, Sezione di Trieste, and Università di Trieste, I-34127 Trieste, Italy \\ ${ }^{79}$ Instituto de Astrofísica de Andalucía, CSIC, E-18080 Granada, Spain \\ ${ }^{80}$ Croatian MAGIC Consortium, Institute R. Bošković, University of Rijeka and University of Split, HR-10000 Zagreb, Croatia \\ ${ }^{81}$ Universitat Autónoma de Barcelona, E-08193 Bellaterra, Spain \\ ${ }^{82}$ Institute for Nuclear Research and Nuclear Energy, BG-1784 Sofia, Bulgaria \\ 83 INAF Osservatorio Astronomico di Brera, I-23807 Merate, Italy \\ ${ }^{84}$ Finnish Centre for Astronomy with ESO (FINCA), University of Turku, FI-21500 Piikiiö, Finland \\ ${ }^{85}$ INAF Osservatorio Astronomico di Trieste, I-34143 Trieste, Italy \\ ${ }^{86}$ Dipartimento di Fisica “Enrico Fermi," Università di Pisa, Pisa I-56127, Italy \\ ${ }^{87}$ INAF, Osservatorio Astronomico di Torino, I-10025 Pino Torinese (TO), Italy \\ ${ }^{88}$ Department of Astronomy, University of Michigan, Ann Arbor, MI 48109-1042, USA \\ ${ }^{89}$ Graduate Institute of Astronomy, National Central University, Jhongli 32054, Taiwan \\ ${ }^{90}$ School of Cosmic Physics, Dublin Institute for Advanced Studies, Dublin, 2, Ireland \\ ${ }^{91}$ Abastumani Observatory, Mt. Kanobili, 0301 Abastumani, Georgia \\ 92 Aalto University Metsähovi Radio Observatory, FIN-02540 Kylmala, Finland \\ ${ }^{93}$ Isaac Newton Institute of Chile, St. Petersburg Branch, St. Petersburg, Russia \\ ${ }^{94}$ Pulkovo Observatory, 196140 St. Petersburg, Russia \\ ${ }^{95}$ Astronomical Institute, St. Petersburg State University, St. Petersburg, Russia \\ ${ }^{96}$ Instituto Nacional de Astrofísica, Óptica y Electrónica, Tonantzintla, Puebla 72840, Mexico \\ ${ }^{97}$ INAF Istituto di Radioastronomia, Sezione di Noto,Contrada Renna Bassa, 96017 Noto (SR), Italy \\ ${ }^{98}$ Physics Department, National University of Ireland Galway, Ireland \\ ${ }^{99}$ Department of Astronomy and Astrophysics, Pennsylvania State University, University Park, PA 16802, USA \\ ${ }^{100}$ Harvard-Smithsonian Center for Astrophysics, Cambridge, MA 02138, USA \\ 101 Astro Space Center of the Lebedev Physical Institute, 117997 Moscow, Russia \\ 102 Department of Physics, Purdue University, West Lafayette, IN 47907, USA \\ ${ }^{103}$ Department of Physics and Astronomy, Brigham Young University, Provo, Utah 84602, USA \\ ${ }^{104}$ INAF Istituto di Radioastronomia, Stazione Radioastronomica di Medicina, I-40059 Medicina (Bologna), Italy \\ ${ }_{105}$ Department of Physics and Astronomy, University of Leicester, Leicester, LE1 7RH, UK \\ ${ }^{106}$ Department of Physics and Astronomy, Whittier College, Whittier, CA, USA \\ 107 Universitat de València, 46010 València, Spain \\ 108 Department of Physics, University of Colorado, Denver, CO 80220, USA \\ ${ }^{109}$ Department of Physics and Astronomy, Pomona College, Claremont CA 91711-6312, USA \\ Received 2010 November 25; accepted 2011 May 19; published 2011 July 15
}

\begin{abstract}
We report on the $\gamma$-ray activity of the high-synchrotron-peaked BL Lacertae object Markarian 421 (Mrk 421) during the first 1.5 years of Fermi operation, from 2008 August 5 to 2010 March 12. We find that the Large Area Telescope (LAT) $\gamma$-ray spectrum above $0.3 \mathrm{GeV}$ can be well described by a power-law function with photon index $\Gamma=1.78 \pm$ 0.02 and average photon flux $F(>0.3 \mathrm{GeV})=(7.23 \pm 0.16) \times 10^{-8} \mathrm{ph} \mathrm{cm}^{-2} \mathrm{~s}^{-1}$. Over this time period, the FermiLAT spectrum above $0.3 \mathrm{GeV}$ was evaluated on seven-day-long time intervals, showing significant variations in the photon flux (up to a factor $\sim 3$ from the minimum to the maximum flux) but mild spectral variations. The variability amplitude at X-ray frequencies measured by RXTE/ASM and Swift/BAT is substantially larger than that in $\gamma$-rays measured by Fermi-LAT, and these two energy ranges are not significantly correlated. We also present the first results from the 4.5 month long multifrequency campaign on Mrk 421, which included the VLBA, Swift, RXTE, MAGIC, the F-GAMMA, GASP-WEBT, and other collaborations and instruments that provided excellent temporal and energy coverage of the source throughout the entire campaign (2009 January 19 to 2009 June 1). During this campaign, Mrk 421 showed a low activity at all wavebands. The extensive multi-instrument (radio to TeV) data set provides an unprecedented, complete look at the quiescent spectral energy distribution (SED) for this source. The broadband SED was reproduced with a leptonic (one-zone synchrotron self-Compton) and a hadronic model (synchrotron proton blazar). Both frameworks are able to describe the average SED reasonably well, implying comparable jet powers but very different characteristics for the blazar emission site.
\end{abstract}

Key words: acceleration of particles - BL Lacertae objects: general - BL Lacertae objects: individual (Mrk 421) galaxies: active - gamma rays: general - radiation mechanisms: non-thermal

Online-only material: color figures

\footnotetext{
${ }^{110}$ Resident at Naval Research Laboratory, Washington, DC 20375, USA.

${ }^{111}$ Royal Swedish Academy of Sciences Research Fellow, funded by a grant from the K. A. Wallenberg Foundation.

${ }^{112}$ Partially supported by the International Doctorate on Astroparticle Physics (IDAPP) program.

${ }^{113}$ NASA Postdoctoral Program Fellow, USA.
}

\section{INTRODUCTION}

Blazars are active galaxies believed to have pairs of relativistic jets flowing in opposite directions closely aligned to our line of

\footnotetext{
${ }^{114}$ Supported by INFN Padova.
} 
sight. Their spectral energy distributions (SEDs) are dominated by beamed jet emission and take the form of two broad nonthermal components, one at low energies, peaking in the radio through optical, and one at high energies, peaking in the $\gamma$-rays. Some blazars have been well monitored for decades and along a wide range of wavelengths. Although there is ample evidence for the electron synchrotron origin of the low-energy bump, the existing data do not allow an unambiguous identification of the radiation mechanism responsible for the high-energy bump. One reason for this is that the high-energy bump is poorly constrained due to the lack of observations at energies between $\sim 0.1 \mathrm{MeV}$ and $0.3 \mathrm{TeV}$. This gap was filled to some extent by EGRET on board the Compton Gamma-Ray Observatory (Hartman et al. 1999). However, its moderate sensitivity and limited observing time precluded detailed cross-correlation studies between $\gamma$-ray and lower-energy wavebands. On the other hand, the current generation of $\mathrm{TeV}$ imaging atmospheric Cherenkov telescopes (IACTs) - the High Energy Stereoscopic System, the Major Atmospheric Gamma Imaging Cherenkov telescope (MAGIC), and the Very Energetic Radiation Imaging Telescope Array System, which have good sensitivity at energies as low as $0.1 \mathrm{TeV}$ - did not start scientific operation until 2004, that is, well after EGRET had stopped operating.

This has changed with the launch of the Fermi Gamma-ray Space Telescope in 2008 June. In science operation since 2008 August, its Large Area Telescope (LAT) instrument (Atwood et al. 2009) views the entire sky in the $20 \mathrm{MeV}$ to greater than $300 \mathrm{GeV}$ range every three hours. The one-year First LAT Active Galactic Nuclei Catalog (1LAC; Abdo et al. 2010b) contains around 600 blazars, a factor of $\sim 10$ greater than EGRET detected during its entire operational lifetime. For the first time, simultaneous observations of Fermi with the latest generation of IACTs can cover the entire highenergy bump. Combining this with simultaneous low-energy observations gives an unprecedented multiwavelength view of these enigmatic objects.

Blazars found in low states are particularly poorly studied. This is due in part to the lower sensitivity of previous instruments, and in part to the fact that multiwavelength monitoring programs, including space-based instruments, are mostly triggered when an object enters a particularly bright state, as observed by ground-based optical telescopes and all-sky monitors such as the RXTE (Bradt et al. 1993) All Sky Monitor (ASM) or the Swift (Gehrels et al. 2004) Burst Alert Telescope (BAT). Having a well-measured low-state SED will be useful for constraining models and as a baseline to which other, flaring states can be compared. This will be crucial for answering many of the questions regarding these objects.

Markarian 421 (Mrk 421; R.A. $=11^{\mathrm{h}} 4^{\mathrm{m}} 27.31$, decl. $=38^{\circ}$ $12^{\prime} 31^{\prime \prime} .8$, J2000, redshift $z=0.031$ ) is a high-synchrotronpeaked (HSP) BL Lac object (according to the classification presented in Abdo et al. (2010c)) that is one of the brightest sources in the extragalactic X-ray/TeV sky. Mrk 421 was actually the first extragalactic object to be discovered as a $\mathrm{TeV}$ emitter (Punch et al. 1992), and one of the fastest varying $\gamma$-ray sources (Gaidos et al. 1996). During the last decade, there were a large number of publications on the very high energy (VHE) $\gamma$-ray spectrum of this source, which has been measured with almost all the existing IACTs (Krennrich et al. 2002; Aharonian et al. 2002, 2003, 2005; Albert et al. 2007a; Acciari et al. 2009). Among other things, we learned that the source shows evidence for a spectral hardening with increasing flux. The SED and the multifrequency correlations of Mrk 421 have also been intensively studied in the past through dedicated multifrequency observations of the source (Katarzyński et al. 2003; Błażejowski et al. 2005; Revillot et al. 2006; Fossati et al. 2008; Horan et al. 2009), which showed a positive but very complex relation between X-rays and VHE $\gamma$-rays, and that a simple one-zone synchrotron self-Compton (SSC) model with an electron distribution parameterized with one or two power laws seemed to describe the collected SED well during the observing campaigns. During a strong flare in 2008 June, the source was also detected with the gamma-ray telescope AGILE and, for the first time, a hint of correlation between optical and TeV energies was reported by Donnarumma et al. (2009).

Despite the large number of publications on Mrk 421, the details of the physical processes underlying the blazar emission are still unknown. The main reasons for this are the sparse multifrequency data during long periods of time, and the moderate sensitivity available in the past to study the $\gamma$-ray emission of this source. In addition, as occurs often with studies of blazars, many of the previous multifrequency campaigns were triggered by an enhanced flux level at X-rays and/or $\gamma$-rays, and hence many of the previous studies of this source are biased toward "high-activity" states, where perhaps distinct physical processes play a dominant role. Moreover, we have very little information from the $\mathrm{MeV}-\mathrm{GeV}$ energy range: nine years of operation with EGRET resulted in only a few viewing periods with a signal significance of barely five standard deviations ( $\sigma$ hereafter; Hartman et al. 1999), which precluded detailed correlation studies with other energy bands.

We took advantage of the new capabilities provided by FermiLAT and the new IACTs, as well as the existing capabilities for observing at X-ray and lower frequencies, and organized a multifrequency (from radio to $\mathrm{TeV}$ ) campaign to observe Mrk 421 over 4.5 months. The observational goal for this campaign was to sample Mrk 421 every two days, which was accomplished at optical, X-ray, and TeV energies whenever the weather and/ or technical operations allowed. Fermi-LAT operated in survey mode and thus the source was constantly observed at $\gamma$-ray energies.

In this paper, we report the overall SED averaged over the duration of the observing campaign. A more in-depth analysis of the multifrequency data set (variability, correlations, and implications) will be given in a forthcoming paper.

This work is organized as follows: In Section 2 we introduce the LAT instrument and report on the data analysis. In Section 3 we report the flux/spectral variability in the $\gamma$-ray range observed by Fermi-LAT during the first 1.5 years of operation, and compare it with the flux variability obtained with $R X T E / \mathrm{ASM}$ and Swift/BAT, which are also all-sky instruments. In Section 4 we report on the spectrum of Mrk 421 measured by Fermi, and Section 5 reports on the overall SED collected during the 4.5 month long multiwavelength campaign organized in 2009 . Section 6 is devoted to SED modeling of the multifrequency data with both a hadronic and a leptonic model, and in Section 7 we discuss the implications of the experimental and modeling results. Finally, we conclude in Section 8.

\section{FERMI-LAT DATA SELECTION AND ANALYSIS}

The Fermi-LAT is a $\gamma$-ray telescope operating from $20 \mathrm{MeV}$ to $>300 \mathrm{GeV}$. The instrument is an array of $4 \times 4$ identical towers, each one consisting of a tracker (where the photons are pair-converted) and a calorimeter (where the energies of the pair-converted photons are measured). The entire instrument is covered with an anticoincidence detector to reject the 

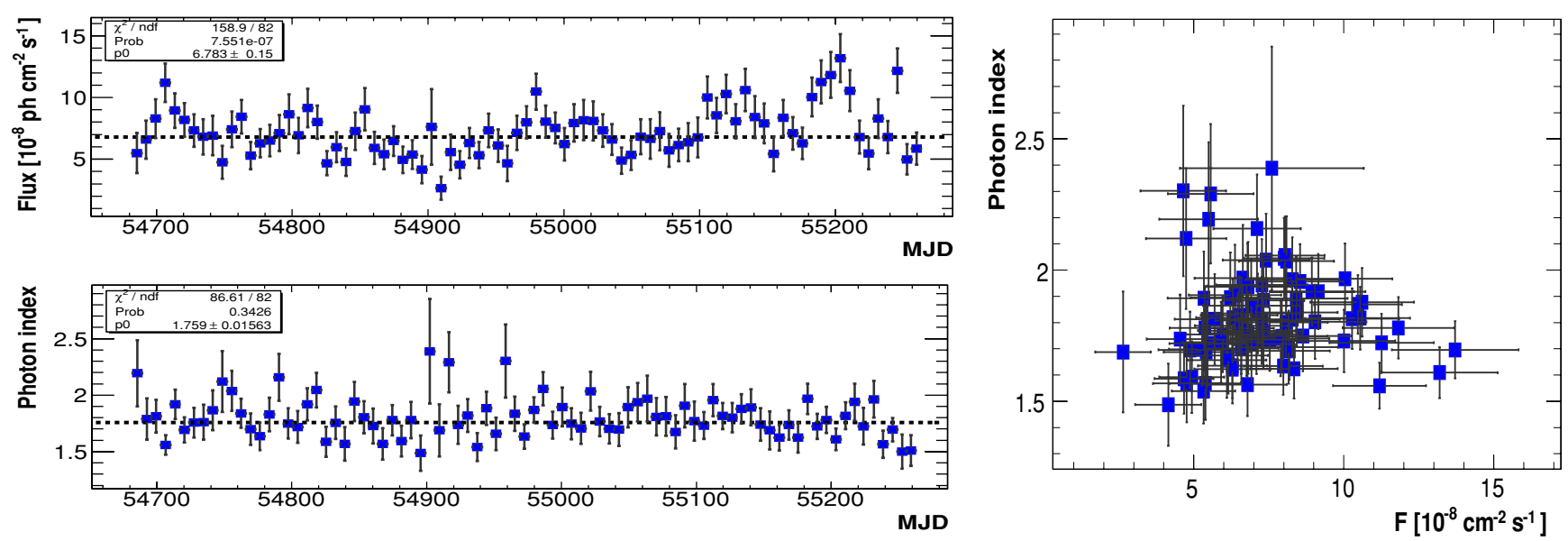

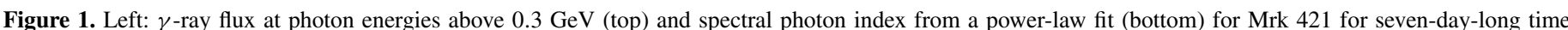

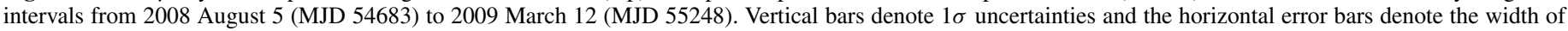
the time interval. The black dashed line and legend show the results from a constant fit to the entire data set. Right: scatter plot of the photon index vs. flux.

(A color version of this figure is available in the online journal.)

charged-particle background. LAT has a large peak effective area $\left(0.8 \mathrm{~m}^{2}\right.$ for $1 \mathrm{GeV}$ photons $)$, an energy resolution typically better than $10 \%$, and a field of view of about $2.4 \mathrm{sr}$ with an angular resolution $\left(68 \%\right.$ containment angle) better than $1^{\circ}$ for energies above $1 \mathrm{GeV}$. Further details on the description of LAT are given by Atwood et al. (2009).

The LAT data reported in this paper were collected from 2008 August 5 (MJD 54683) to 2010 March 12 (MJD 55248). During this time, the Fermi-LAT instrument operated almost entirely in survey mode. The analysis was performed with the Science Tools software package version v9r15p6. Only events having the highest probability of being photons, those in the "diffuse" class, were used. The LAT data were extracted from a circular region with a $10^{\circ}$ radius centered at the location of Mrk 421. The spectral fits were performed using photon energies greater than $0.3 \mathrm{GeV}$, where the effective area of the instrument is large $\left(>0.5 \mathrm{~m}^{2}\right)$ and the angular resolution relatively good $(68 \%$ containment angle smaller than $2^{\circ}$ ). The spectral fits using energies above $0.3 \mathrm{GeV}$ are less sensitive to possible contamination from non-accounted (transient) neighboring sources, and have smaller systematic errors, at the expense of reducing somewhat the number of photons from the source. In addition, a cut on the zenith angle $\left(<105^{\circ}\right)$ was also applied to reduce contamination from the Earth limb $\gamma$-rays, which are produced by cosmic rays interacting with the upper atmosphere.

The background model used to extract the $\gamma$-ray signal includes a Galactic diffuse emission component and an isotropic component. The model that we adopted for the Galactic component is given by the file gll_iem_v02.fit, and the isotropic component, which is the sum of the extragalactic diffuse emission and the residual charged particle background, is parameterized by the file isotropic_iem_v02.115 The normalization of both components in the background model was allowed to vary freely during the spectral point fitting. The spectral analyses (from which we derived spectral fits and photon fluxes) were performed with the post-launch instrument response functions P6_V3_DIFFUSE using an unbinned maximum likelihood method. The systematic uncertainties in the flux were estimated

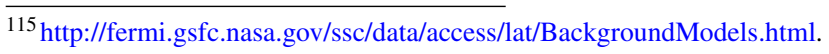

as $10 \%$ at $0.1 \mathrm{GeV}, 5 \%$ at $560 \mathrm{MeV}$ and $20 \%$ at $10 \mathrm{GeV}$ and above. $^{116}$

\section{FLUX AND SPECTRAL VARIABILITY}

The sensitivity of Fermi-LAT is sufficient to accurately monitor the $\gamma$-ray flux of Mrk 421 on short timescales (a few days). ${ }^{117}$ The measured $\gamma$-ray flux above $0.3 \mathrm{GeV}$ and the photon index from a power-law (PL) fit are shown in Figure 1. The data span the time from 2008 August 5 (MJD 54683) to 2009 March 12 (MJD 55248) and they are binned on time intervals of 7 days. The Test Statistic (TS) values ${ }^{118}$ for the 81 time intervals are typically well in excess of 100 $(\sim 10 \sigma)$. The number of intervals with TS $<100$ is only nine $(11 \%)$. The lowest TS value is 30 , which occurs for the time interval MJD 54899-54906. This low signal significance is due to the fact that the Fermi-LAT instrument did not operate during the time interval MJD 54901-54905 ${ }^{119}$ and hence only three out of the seven days of the interval contain data. The second lowest TS value is 40, which occurred for the time interval 54962-54969. During the first 19 months of Fermi operation, Mrk 421 showed relatively mild $\gamma$-ray flux variations, with the lowest photon flux $F(>0.3 \mathrm{GeV})=$ $(2.6 \pm 0.9) \times 10^{-8} \mathrm{~cm}^{-2} \mathrm{~s}^{-1}$ (MJD 54906-54913; TS = 53) and the highest $F(>0.3 \mathrm{GeV})=(13.2 \pm 1.9) \times 10^{-8} \mathrm{~cm}^{-2} \mathrm{~s}^{-1}$ (MJD 55200-55207; TS = 355). A constant fit to the flux points from Figure 1 gave a $\chi^{2}=159$ for 82 degrees of freedom (probability that the flux was constant is $8 \times 10^{-7}$ ), hence indicating the existence of statistically significant flux variability. On the other hand, the photon index measured in seven-day-long time intervals is statistically compatible with being constant, as indicated by the results of the constant fit to all the photon index values, which gave $\chi^{2}=87$ for 82 degrees of freedom (NDF; probability of no variability is 0.34 ). The scatter

\footnotetext{
${ }^{116}$ See http://fermi.gsfc.nasa.gov/ssc/data/analysis/LAT_caveats.html.

${ }^{117}$ The number of photons from Mrk 421 (above $0.3 \mathrm{GeV}$ ) detected by LAT in one day is typically about six.

${ }^{118}$ The Test Statistic TS $=2 \Delta \log$ (likelihood) between models with and without the source is a measure of the probability of having a point $\gamma$-ray source at the location specified. The TS value is related to the significance of the signal (Mattox et al. 1996).

${ }^{119}$ The LAT did not operate during the time interval MJD 54901-54905 due to an unscheduled shutdown.
} 

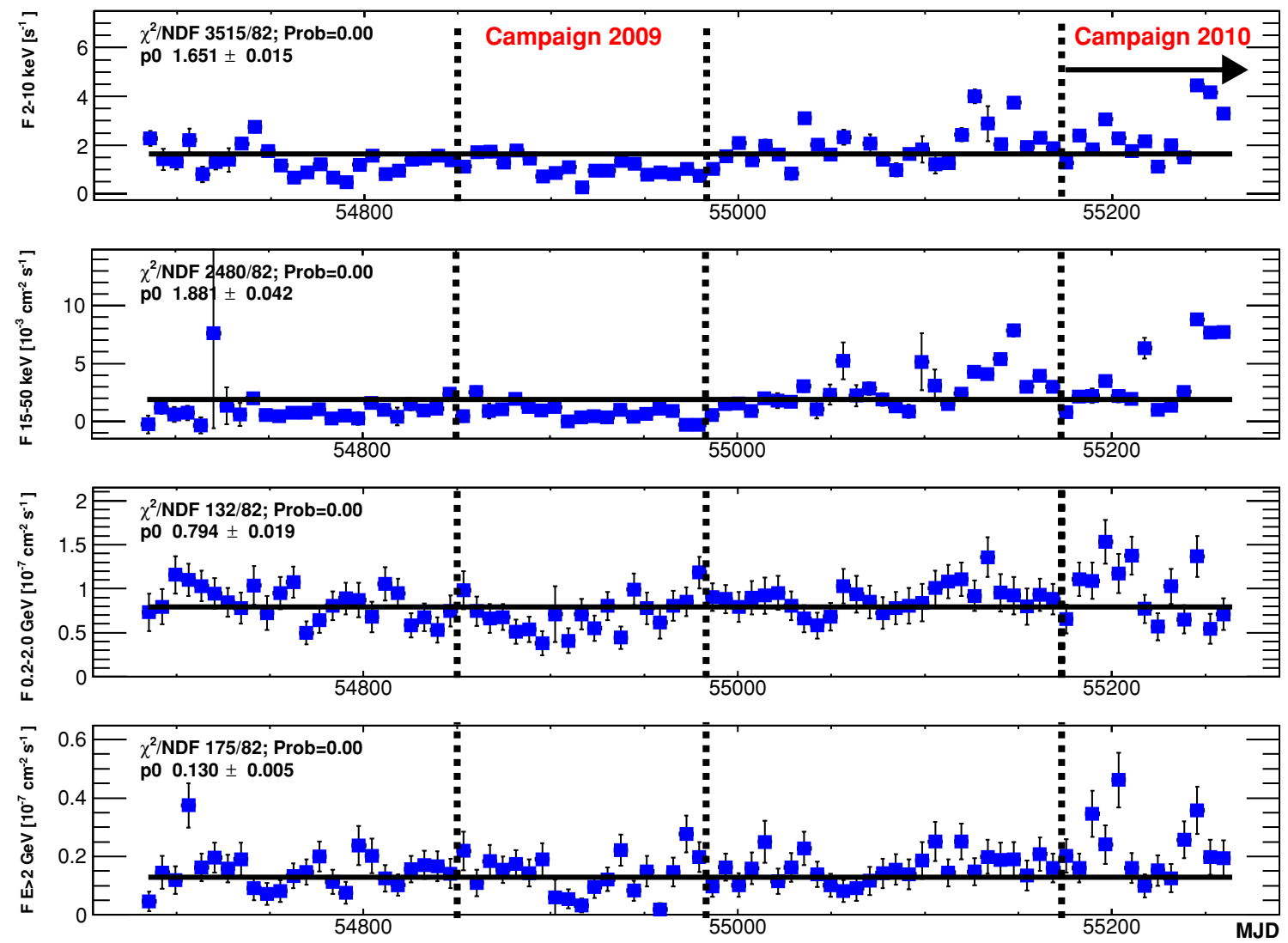

Figure 2. Multifrequency light curves of Mrk 421 with seven-day-long time bins obtained with three all-sky-monitoring instruments: RXTE/ASM (2-10 keV, top), Swift/BAT (15-50 keV, second from top), and Fermi-LAT for two different energy ranges ( $0.2-2 \mathrm{GeV}$, third from top, and $>2 \mathrm{GeV}$, bottom). The light curves cover the period from 2008 August 5 (MJD 54683) to 2009 March 12 (MJD 55248). Vertical bars denote $1 \sigma$ uncertainties and horizontal error bars show the width of the time interval. The black dashed lines and legends show the results from constant fits to the entire data set. The vertical dashed lines denote the time intervals with the extensive multifrequency campaigns during the 2009 and 2010 seasons.

(A color version of this figure is available in the online journal.)

plot with Flux versus Index in Figure 1 shows that there is no obvious relation between these two quantities. We quantified the correlation as prescribed in Edelson \& Krolik (1988), obtaining a discrete correlation function $\mathrm{DCF}=0.06 \pm 0.11$ for a time lag of zero.

It is interesting to compare the $\gamma$-ray fluxes measured by Fermi with those historical ones recorded by EGRET. From the third EGRET catalog (Hartman et al. 1999), one can see that the highest and lowest significantly measured $(\mathrm{TS}>25)$ photon fluxes are $F^{\mathrm{Max}}(>0.1 \mathrm{GeV})=(27.1 \pm 6.9) \times 10^{-8} \mathrm{~cm}^{-2} \mathrm{~s}^{-1}$ $(\mathrm{TS}=32)$ and $F^{\mathrm{Min}}(>0.1 \mathrm{GeV})=(10.9 \pm 2.8) \times 10^{-8} \mathrm{~cm}^{-2} \mathrm{~s}^{-1}$ (TS $=26)$, respectively, where $F(>0.1 \mathrm{GeV})$ is the flux above $0.1 \mathrm{GeV}$. These values do not deviate by more than $2 \sigma$ from the P1234 average, $F(>0.1 \mathrm{GeV})=(13.8 \pm 1.8) \times 10^{-8} \mathrm{~cm}^{-2} \mathrm{~s}^{-1}$ (TS $=100)$, and hence EGRET did not detect significant variability in the flux from Mrk 421. We can easily obtain the Fermi $F(>0.1 \mathrm{GeV})$ fluxes by using the flux $(\mathrm{F})$ index $(\Gamma)$ values reported in Figure $1(E>0.3 \mathrm{GeV}): F(>0.1 \mathrm{GeV})=$ $F(>0.3 \mathrm{GeV}) \times(0.3 / 0.1)^{\Gamma-1}$. Applying this simple formalism one gets, for the maximum and minimum fluxes from Figure $1, F^{\mathrm{Max}}(>0.1 \mathrm{GeV})=(25.7 \pm 4.7) \times 10^{-8} \mathrm{~cm}^{-2} \mathrm{~s}^{-1}$ and $F^{\mathrm{Min}}(>0.1 \mathrm{GeV})=(5.6 \pm 2.4) \times 10^{-8} \mathrm{~cm}^{-2} \mathrm{~s}^{-1}$, respectively. The maximum flux measured by EGRET and LAT are similar, although the minimum fluxes are not. LAT's larger effective area compared to EGRET permits detection of lower $\gamma$-ray fluxes. In any case, the EGRET and LAT fluxes are comparable, which may indicate that Mrk 421 is not as variable in the
$\mathrm{MeV} / \mathrm{GeV}$ range as at other wavelengths, particularly X-rays and TeV $\gamma$-rays (e.g., Wagner 2008).

The Fermi-LAT capability for constant source monitoring is nicely complemented at X-ray energies by $R X T E / \mathrm{ASM}$ and Swift/BAT, the two other all-sky instruments that can probe the X-ray activity of Mrk 421 in seven-day-long time intervals. Figure 2 shows the measured fluxes by ASM in the energy range $2-10 \mathrm{keV}$, by BAT at $15-50 \mathrm{keV}$, and by LAT in two different energy bands: $0.2-2 \mathrm{GeV}$ (low energy) and $>2 \mathrm{GeV}$ (high energy). ${ }^{120}$ The low and high Fermi-LAT energy bands were chosen (among other reasons) to produce comparable flux errors. This might seem surprising at first glance, given that the number of detected photons in the low energy band is about five times larger than in the high energy band (for a differential energy spectrum parameterized by a PL with photon index of 1.8, which is the case of Mrk 421). Hence the number of detected $\gamma$-rays decreases from about 50 down to about 10 for time intervals of seven days. The main reason for having comparable flux errors in these two energy bands is that the diffuse background, which follows a PL with index 2.4 for the high galactic latitude of Mrk 421, is about 25 times smaller in the high energy band. Consequently, signal to noise $\left.\sim N_{S} / \sqrt{(} N_{B}\right)$ remains approximately equal.

\footnotetext{
${ }^{120}$ The fluxes depicted in the light curves were computed fixing the photon index to 1.78 (average index during the first 1.5 years of Fermi operation) and fitting only the normalization factor of the PL function.
} 

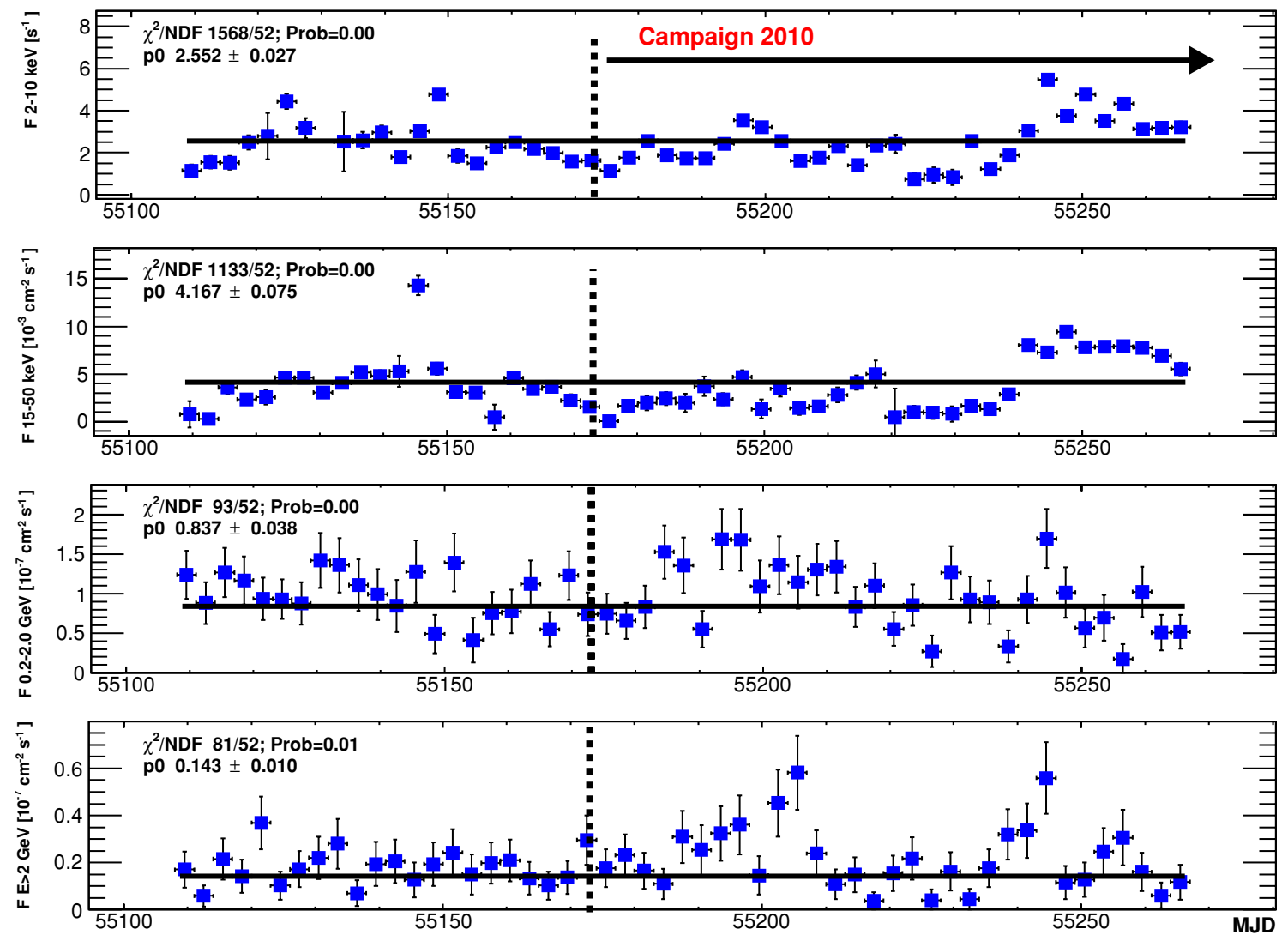

Figure 3. Multifrequency light curves of Mrk 421 with three-day-long time bins obtained with three all-sky-monitoring instruments: RXTE/ASM (2-10 keV, top), Swift/BAT (15-50 keV, second from top), and Fermi-LAT for two different energy ranges ( $0.2-2 \mathrm{GeV}$, third from top, and $>2 \mathrm{GeV}$, bottom). The light curves cover the period from 2009 October 4 to 2010 March 12. Vertical bars denote $1 \sigma$ uncertainties and horizontal error bars show the width of the time interval. The black dashed lines and legends show the results from constant fits to the entire data set. The vertical dashed lines denote the beginning of the extensive multifrequency campaign on Mrk 421 during the 2010 season.

(A color version of this figure is available in the online journal.)

We do not see variations in the LAT hardness ratio (i.e., $F(>2 \mathrm{GeV}) / F(0.2-2 \mathrm{GeV})$ with the $\gamma$-ray flux, but this is limited by the relatively large uncertainties and the low $\gamma$-ray flux variability during this time interval. The data from RXTE/ASM were obtained from the ASM Web site. ${ }^{121}$ We filtered out the data according to the provided prescription on the ASM Web site, and made a weighted average of all the dwells (scan/rotation of the ASM Scanning Shadow Cameras lasting $90 \mathrm{~s})$ from the seven-day-long time intervals defined for the Fermi data. The data from Swift/BAT were gathered from the BAT Web site. ${ }^{122}$ We retrieved the daily averaged BAT values and produced a weighted average for all the seven-day-long time intervals defined for the Fermi data.

The X-ray flux from Mrk 421 was $\sim 1.7$ counts $\mathrm{s}^{-1}$ in ASM and $\sim 1.9 \times 10^{-3}$ counts s $\mathrm{s}^{-1} \mathrm{~cm}^{-2}$ in BAT. These fluxes correspond to $\sim 22 \mathrm{mCrab}$ in ASM $\left(1 \mathrm{Crab}=75\right.$ counts $\left.\mathrm{s}^{-1}\right)$ and $9 \mathrm{mCrab}$ in BAT $\left(1 \mathrm{Crab}=0.22\right.$ counts s $\left.\mathrm{cm}^{-1}\right)$, although given the recent reports on flux variability from the Crab Nebula (see Wilson-Hodge et al. 2011; Abdo et al. 2011a; Tavani et al. 2011), the flux from the Crab Nebula is not a good absolute standard candle any longer and hence those numbers need to be taken with caveats. One may note that the X-ray activity was rather low during the first year of Fermi operation. The X-ray activity increased around MJD 54990 and then increased even more around MJD 55110. The $\gamma$-ray activity seemed to follow

\footnotetext{
${ }^{121}$ See http://xte.mit.edu/ASM_lc.html.

${ }^{122} \mathrm{See}$ http://swift.gsfc.nasa.gov/docs/swift/results/transients/.
}

some of the X-ray activity, but the variations in the $\gamma$-ray range are substantially smoother than those observed in X-rays.

Figure 3 shows the same light curves as Figure 2, but only during the period of time after MJD 55110 (when Mrk 421 showed high X-ray activity) with a time bin of only three days. During this time period the ASM and BAT flux (integrated over three days) went beyond 5 counts $\mathrm{s}^{-1}$ and $8 \times 10^{-3}$ counts $\mathrm{s}^{-1} \mathrm{~cm}^{-2}$, respectively, which implies a flux increase by a factor of five to eight with respect to the average fluxes during the first year. It is worth noting that these large flux variations do not have a counterpart at $\gamma$-ray energies measured by Fermi-LAT. The MeV/GeV flux measured by LAT remained roughly constant, with the exception of a flux increase by a factor of about two for the time intervals around MJD 55180-55210 and around MJD 55240-55250, which was also seen by RXTE/ ASM and (to some extent) by Swift/BAT.

We quantified the correlation among the light curves shown in Figures 2 and 3 following the prescription from Edelson \& Krolik (1988). The results are shown in Table 1 for a time lag of zero, which is the one giving the largest DCF values. There is no indication of correlated activity at positive/negative time lags in the DCF versus time plot for any of the used $\mathrm{X}$-ray $/ \gamma$-ray bands. The advantage of using the DCF instead of the Pearson's correlation coefficient is that the latter does not consider the error in the individual flux points, while the former does. In this particular situation it is relevant to consider these errors because they are sometimes comparable 
Table 1

Discrete Correlation Function (DCF)

\begin{tabular}{|c|c|c|c|c|c|c|}
\hline Interval & ASM-BAT & $\mathrm{ASM}^{-\mathrm{LAT}_{<2 \mathrm{GeV}}}$ & $\mathrm{ASM}^{-\mathrm{LAT}_{>}} 2 \mathrm{GeV}$ & BAT-LAT $<2 \mathrm{GeV}$ & BAT-LAT $_{>2 \mathrm{GeV}}$ & $\mathrm{LAT}_{<2 \mathrm{GeV}}-\mathrm{LAT}_{>2 \mathrm{GeV}}$ \\
\hline 7 days & $0.73 \pm 0.20$ & $0.28 \pm 0.15$ & $0.35 \pm 0.14$ & $0.20 \pm 0.13$ & $0.26 \pm 0.13$ & $0.31 \pm 0.14$ \\
\hline 3 days & $0.65 \pm 0.13$ & $0.01 \pm 0.18$ & $0.15 \pm 0.19$ & $-0.03 \pm 0.13$ & $0.01 \pm 0.13$ & $0.29 \pm 0.17$ \\
\hline
\end{tabular}

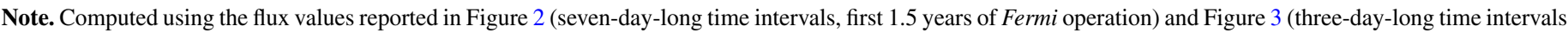

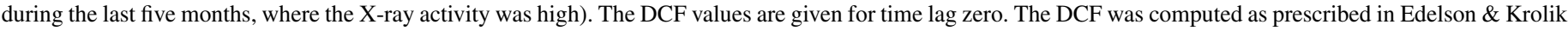
(1988).

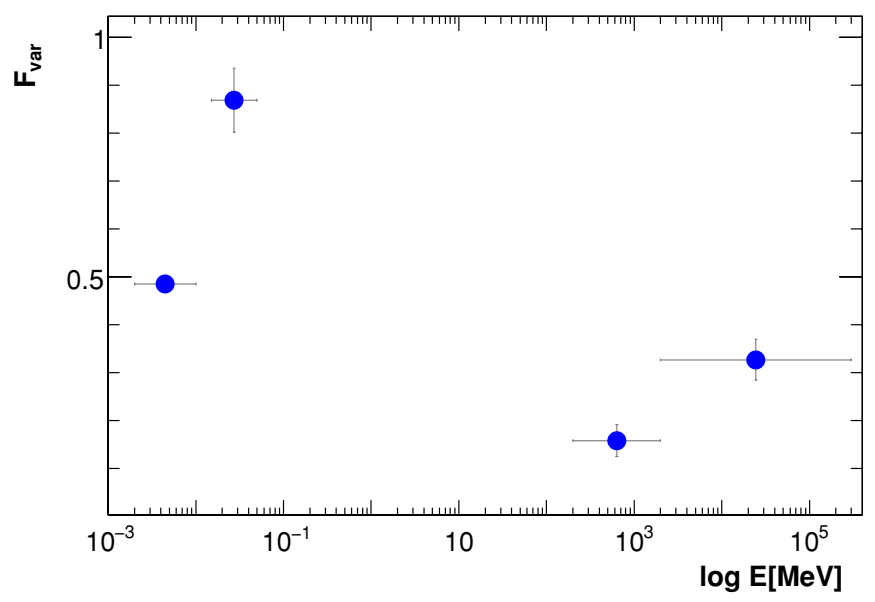

Figure 4. Fractional variability parameter for 1.5 year data (2008 August 5-2009 March 12) from three all-sky-monitoring instruments: RXTE/ASM (2-10 keV, first), Swift/BAT (15-50 keV, second) and Fermi-LAT for two energy ranges $0.2-2 \mathrm{GeV}$ and $2-300 \mathrm{GeV}$. The fractional variability was computed according to Vaughan et al. (2003) using the light curves from Figure 2. Vertical bars denote $1 \sigma$ uncertainties and horizontal bars indicate the width of each energy bin.

(A color version of this figure is available in the online journal.)

to the magnitude of the measured flux variations. The main result is a clear $\left(\mathrm{DCF} / \mathrm{DCF}_{\text {error }} \sim 4\right)$ correlation between $\mathrm{ASM}$ and BAT, while there is no indication of X-ray $/ \gamma$-ray correlation $\left(\mathrm{DCF} / \mathrm{DCF}_{\text {error }} \lesssim 2\right.$ ). The correlation between the Fermi-LAT fluxes below and above $2 \mathrm{GeV}$ is not significant $(\mathrm{DCF}=0.31 \pm 0.14)$, which is probably due to the low variability at $\gamma$-rays, together with the relatively large flux errors for the individual seven-day-long and three-day-long time intervals.

We followed the prescription given in Vaughan et al. (2003) to quantify the flux variability by means of the fractional variability parameter, $F_{\text {var }}$, as a function of energy. In order to account for the individual flux measurement errors $\left(\sigma_{\mathrm{err}, i}\right)$, we used the "excess variance" (Nandra et al. 1997; Edelson et al. 2002) as an estimator of the intrinsic source variance. This is the variance after subtracting the expected contribution from measurement errors. For a given energy range, the $F_{\text {var }}$ is calculated as

$$
F_{\mathrm{var}}=\sqrt{\frac{S^{2}-\left\langle\sigma_{\mathrm{err}}^{2}\right\rangle}{\langle F\rangle^{2}}},
$$

where $\langle F\rangle$ is the mean photon flux, $S$ the standard deviation of the $N$ flux points, and $\left\langle\sigma_{\text {err }}^{2}\right\rangle$ the average mean square error, all determined for a given energy bin.

Figure 4 shows the derived $F_{\text {var }}$ values for the four energy ranges and the time window covered by the light curves shown in Figure 2. The fractional variability is significant for all energy ranges, with the $\mathrm{X}$-rays having a substantially higher variability than the $\gamma$-rays.
It is interesting to note that while the PL photon index variations from Figure 1 were not statistically significant $\left(\chi^{2} / N D F=87 / 82\right)$, Figure 4 shows that the fractional variability for photon energies above $2 \mathrm{GeV}$ is higher than that below $2 \mathrm{GeV}$; specifically, $F_{\mathrm{var}}(E<2 \mathrm{GeV})=0.16 \pm 0.04$ while $F_{\text {var }}(E>2 \mathrm{GeV})=0.33 \pm 0.04$. This apparent discrepancy between the results reported in Figure 1 and the ones reported in Figure 4 (produced with the flux points from Figure 2) might be due to the fact that, on timescales of seven days, the photons below $2 \mathrm{GeV}$ dominate the determination of the PL photon index in the unbinned likelihood fit. In other words, the source is bright enough in the energy range $0.3-2 \mathrm{GeV}$ such that the (relatively few) photons above $2 \mathrm{GeV}$ do not have a large (statistical) weight in the computation of the PL photon index. Consequently, we are more sensitive to spectral variations when doing the analysis separately for these two energy ranges.

One may also note that, besides the larger variability in the Fermi fluxes above $2 \mathrm{GeV}$ with respect to those below $2 \mathrm{GeV}$, the variability in the BAT fluxes $(15-50 \mathrm{keV})$ is also higher than that of ASM (2-10 keV). The implications of this experimental result will be further discussed in Section 7.3, in light of the modeling results presented in Section 6.2.

\section{SPECTRAL ANALYSIS UP TO $400 \mathrm{GeV}$}

The LAT instrument allows one to accurately reconstruct the photon energy over many orders of magnitude. Figure 5 shows the spectrum of Mrk 421 in the energy range $0.1-400 \mathrm{GeV}$. This is the first time that the spectrum of Mrk 421 can be studied with this level of detail over this large a fraction of the electromagnetic spectrum, which includes the previously unexplored energy range $10-100 \mathrm{GeV}$. The spectrum was computed using the analysis procedures described in Section 2. In order to reduced systematics, the spectral fit was performed using photon energies greater than $0.3 \mathrm{GeV}$, where the LAT instrument has good angular resolution and large effective area. The black line in Figure 5 is the result of a fit with a single PL function over the energy range $0.3-400 \mathrm{GeV}$, and the red contour is the $68 \%$ uncertainty of the fit. The data are consistent with a pure PL function with a photon index of $1.78 \pm 0.02$. The black data points are the result of performing the analysis on differential energy ranges ( 2.5 bins per decade of energy). ${ }^{123}$ The points are well within $1 \sigma-2 \sigma$ from the fit to the overall spectrum (black line), which confirms that the entire Fermi spectrum is consistent with a pure PL function.

However, it is worth noticing that the error bars at the highest energies are relatively large due to the low photon count. In the

\footnotetext{
${ }^{123}$ Because of the analysis being carried out in small energy ranges, we fixed the spectral index to 1.78 , which is the value obtained when fitting the entire energy range. We repeated the same procedure fixing the photon indices to 1.5 and 2.0, and found no significant change. Therefore, the results from the differential energy analysis are not sensitive to the selected photon index used in the analysis.
} 


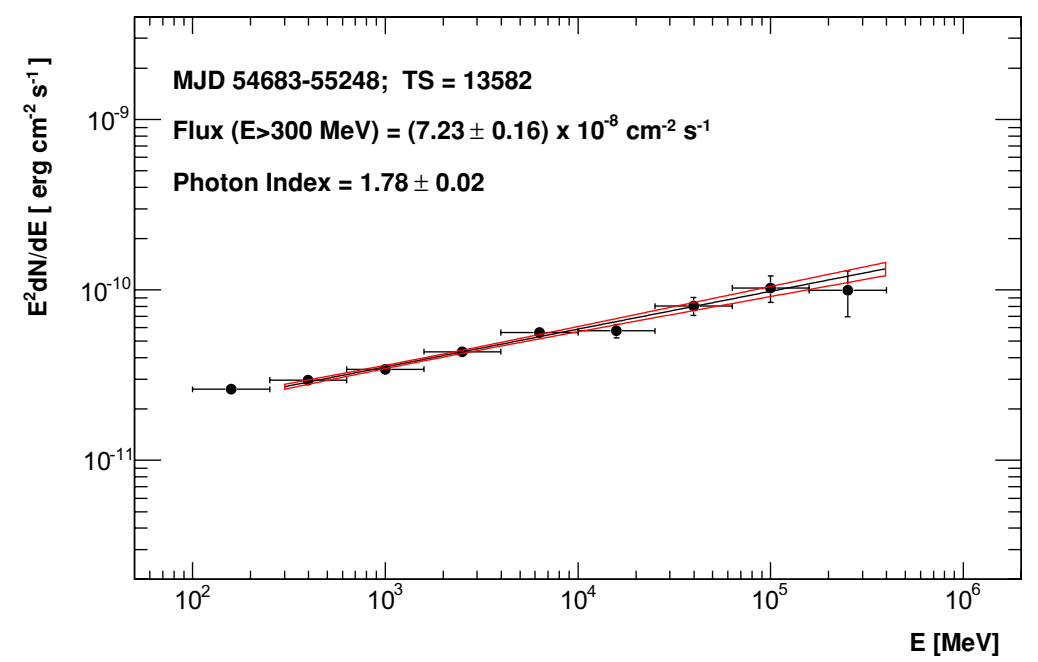

Figure 5. Fermi spectrum of Mrk 421 during the period from 2008 August 5 to 2010 February 20. The black line is the likelihood PL fit, the red contour is the $68 \%$ uncertainty of the fit, and the black data points show the energy fluxes computed on differential energy ranges. The inlay summarizes the unbinned likelihood PL fit in the energy range $0.3-400 \mathrm{GeV}$.

(A color version of this figure is available in the online journal.)

energy bins $60-160 \mathrm{GeV}$ and $160-400 \mathrm{GeV}$, the predicted (by the model for Mrk 421) numbers of photons detected by LAT are 33 and 11, respectively. Even though the low background makes those signals very significant (TS values of 562 and 195 , respectively), the statistical uncertainties in the energy flux values are naturally large and hence they could hide a potential turnover in the spectrum of Mrk 421 at around $100 \mathrm{GeV}$. Indeed, when performing the likelihood analysis on LAT data above $100 \mathrm{GeV}$, one obtains a photon flux above $100 \mathrm{GeV}$ of $(5.6 \pm 1.1) \times 10^{-10} \mathrm{ph} \mathrm{cm}^{-2} \mathrm{~s}^{-1}$ with a photon index of $2.6 \pm 0.6$, which might suggest a turnover in the spectrum, consistent with the TeV spectra determined by past observations with IACTs (Krennrich et al. 2002; Aharonian et al. 2003, 2005; Albert et al. 2007a). In order to make a statistical evaluation of this possibility, the LAT spectrum (in the range $0.3-400 \mathrm{GeV}$ ) was fit with a broken power law (BPL) function, obtaining the indices of $1.77 \pm 0.02$ and $2.9 \pm 1.0$ below and above the break energy of $182 \pm 39 \mathrm{GeV}$, respectively. The likelihood ratio of the BPL and the PL gave 0.7, which, given the two extra degrees of freedom for the BPL function, indicates that the BPL function is not statistically preferred over the PL function. Therefore, the statistical significance of the LAT data above $100 \mathrm{GeV}$ is not sufficiently high to evaluate the potential existence of a break (peak) in the spectrum.

\section{SPECTRAL ENERGY DISTRIBUTION OF MRK 421 DURING THE 4.5 MONTH LONG MULTIFREQUENCY CAMPAIGN FROM 2009}

As mentioned in Section 1, we organized a multifrequency (from radio to $\mathrm{TeV}$ photon energies) campaign to monitor Mrk 421 during a time period of 4.5 months. The observing campaign started on 2009 January 19 (MJD 54850) and finished on 2009 June 1 (MJD 54983). The observing strategy for this campaign was to sample the broadband emission of Mrk 421 every two days, which was accomplished at optical, X-ray, and $\mathrm{TeV}$ energies when the weather and/or technical limitations allowed. The main goal of this project was to collect an extensive multifrequency data set that is simultaneous and representative of the average/typical SED from Mrk 421. Such a data set can provide additional constraints that will allow us to refine the emission models, which in turn will provide new insights into the processes related to the particle acceleration and radiation in this source. In this section we describe the source coverage during the campaign, the data analysis for several of the participating instruments, and finally we report on the averaged SED resulting from the whole campaign.

\subsection{Details of the Campaign: Participating Instruments and Temporal Coverage}

The list of all the instruments that participated in the campaign is reported in Table 2, and the scheduled observations can be found online. ${ }^{124}$ We note that in some cases the planned observations could not be performed due to bad observing conditions, while on other occasions the observations were performed but the data could not be properly analyzed due to technical problems or rapidly changing weather conditions. Figure 6 shows the time coverage as a function of the energy range for the instruments/observations used to produce the SED shown in Figure 8. Apart from the unprecedented energy coverage (including, for the first time, the GeV energy range from FermiLAT), the source was sampled very uniformly with the various instruments participating in the campaign and, consequently, it is reasonable to consider the SED constructed below as the actual average (typical) SED of Mrk 421 during the time interval covered by this multifrequency campaign. The largest non-uniformity in the sampling of the source comes from the Cherenkov telescopes, which are the instruments most sensitive to weather conditions. Moreover, while there are many radio/optical instruments spread all over the globe, in this observing campaign only two Cherenkov telescope observatories participated, namely MAGIC and the Fred Lawrence Whipple Observatory. Hence, the impact of observing conditions was more important to the coverage at the VHE $\gamma$-ray energies. During the time interval MJD 54901-54905, the Fermi satellite did not operate due to a spacecraft technical problem. The lack of Fermi-LAT data during this period is clearly seen in Figure 6.

We note that Figure 6 shows the MAGIC and Whipple coverage in VHE $\gamma$-ray energies, but only the MAGIC observations

\footnotetext{
${ }^{124}$ See https://confluence.slac.stanford.edu/display/GLAMCOG/Campaign +on+Mrk421+(Jan+2009+to+May+2009) maintained by D. Paneque.
} 


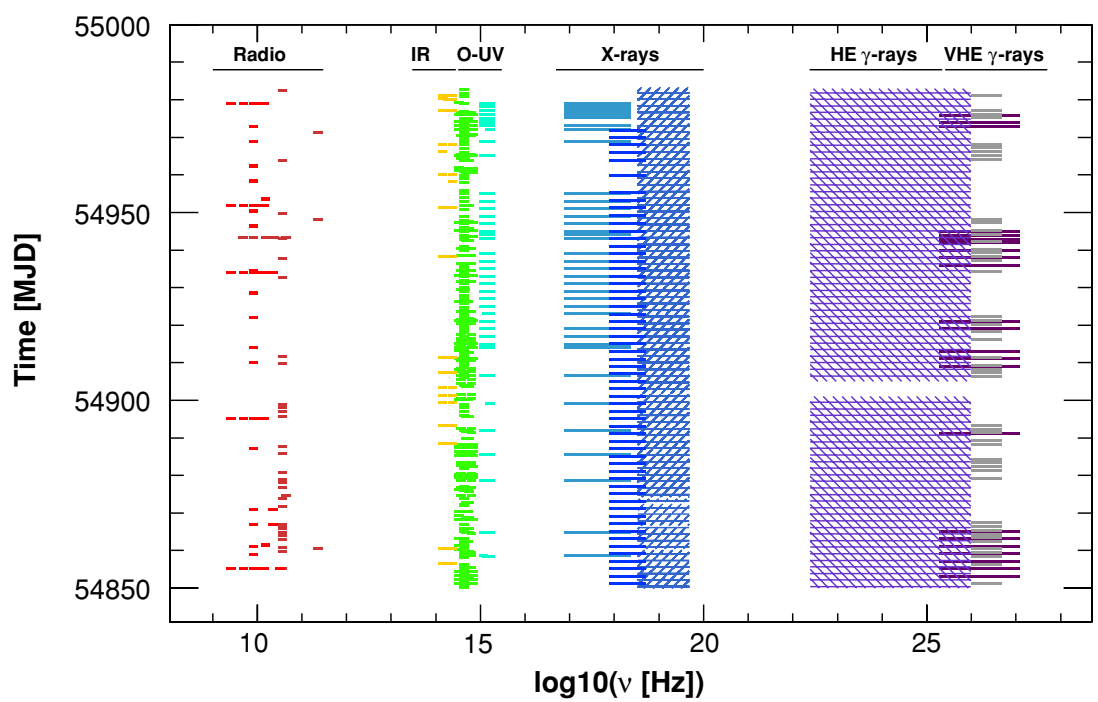

Figure 6. Time and energy coverage during the multifrequency campaign. For the sake of clarity, the minimum observing time displayed in the plot was set to half a day.

Table 2

List of Instruments Participating in the Multifrequency Campaign and Used in the Compilation of the SED Shown in Figure 8

\begin{tabular}{|c|c|c|}
\hline Instrument/Observatory & Energy Range Covered & Web Site \\
\hline MAGIC & $0.08-5.0 \mathrm{TeV}$ & http://wwwmagic.mppmu.mpg.de/ \\
\hline Whipple $^{\mathrm{a}}$ & $0.4-2.0 \mathrm{TeV}$ & http://veritas.sao.arizona.edu/content/blogsection/6/40/ \\
\hline Fermi-LAT & $0.1-400 \mathrm{GeV}$ & http://www-glast.stanford.edu/index.html \\
\hline$R X T E / \mathrm{PCA}$ & $3-32 \mathrm{keV}$ & http://heasarc.gsfc.nasa.gov/docs/xte/rxte.html \\
\hline Swift/XRT & $0.3-9.6 \mathrm{keV}$ & http://heasarc.gsfc.nasa.gov/docs/swift/swiftsc.html \\
\hline Swift/UVOT & UVW1, UVM2, UVW2 & http://heasarc.gsfc.nasa.gov/docs/swift/swiftsc.html \\
\hline $\begin{array}{l}\text { Roque de los Muchachos (KVA; through GASP-WEBT } \\
\text { program) }\end{array}$ & $R$ band & http://www.oato.inaf.it/blazars/webt/ \\
\hline St. Petersburg (through GASP-WEBT program) & $R$ band & http://www.oato.inaf.it/blazars/webt/ \\
\hline Talmassons (through GASP-WEBT program) & $R$ band & http://www.oato.inaf.it/blazars/webt/ \\
\hline Valle d'Aosta (through GASP-WEBT program) & $R$ band & http://www.oato.inaf.it/blazars/webt/ \\
\hline GRT & $V, R, B, I$ bands & http://asd.gsfc.nasa.gov/Takanori.Sakamoto/GRT/index.html \\
\hline WIRO & $J, K$ bands & http://physics.uwyo.edu/chip/wiro/wiro.html \\
\hline SMA & $225 \mathrm{GHz}$ & http://sma1.sma.hawaii.edu/ \\
\hline VLBA & $4.8,8.3,15.4,23.8,43.2 \mathrm{GHz}$ & http://www.vlba.nrao.edu/ \\
\hline Noto & $8.4,22.3 \mathrm{GHz}$ & http://www.noto.ira.inaf.it/ \\
\hline Metsähovi (through GASP-WEBT program) & $37 \mathrm{GHz}$ & http://www.metsahovi.fi/ \\
\hline VLBA (through MOJAVE program) & $15 \mathrm{GHz}$ & http://www.physics.purdue.edu/MOJAVE/ \\
\hline OVRO & $15 \mathrm{GHz}$ & http://www.ovro.caltech.edu/ \\
\hline Medicina & $8.4 \mathrm{GHz}$ & http://www.med.ira.inaf.it/index_EN.htm \\
\hline UMRAO (through GASP-WEBT program) & $4.8,8.0,14.5 \mathrm{GHz}$ & http://www.oato.inaf.it/blazars/webt/ \\
\hline RATAN-600 & $2.3,4.8,7.7,11.1,22.2 \mathrm{GHz}$ & http://w0.sao.ru/ratan/ \\
\hline Effelsberg (through F-GAMMA program) & $2.6,4.6,7.8,10.3,13.6,21.7,31 \mathrm{GHz}$ & http://www.mpifr-bonn.mpg.de/div/effelsberg/index_e.html/ \\
\hline
\end{tabular}

Notes. The energy range shown in Column 2 is the actual energy range covered during the Mrk 421 observations, and not the instrument nominal energy range, which might only be achievable for bright sources and in excellent observing conditions.

a The Whipple spectra were not included in Figure 8. See the text for further comments.

were used to produce the spectra shown in Figure 8. The more extensive, but less sensitive, Whipple data (shown as gray boxes in Figure 6) were primarily taken to determine the light curve (Pichel et al. 2009) and a re-optimization was required to derive the spectrum, which will be reported elsewhere.
In the following paragraphs we briefly discuss the procedures used in the analysis of the instruments participating in the campaign. The analysis of the Fermi-LAT data was described in Section 2 and the results obtained will be described in detail in Section 5.2. 


\subsubsection{Radio Instruments}

Radio data were taken for this campaign from single-dish telescopes, one millimeter interferometer, and one very long baseline interferometry (VLBI) array, at frequencies between 2.6 GHz and $225 \mathrm{GHz}$ (see Table 2). The single-dish telescopes were the Effelsberg $100 \mathrm{~m}$ radio telescope, the Medicina $32 \mathrm{~m}$ radio telescope, the Metsähovi $14 \mathrm{~m}$ radio telescope, the Noto $32 \mathrm{~m}$ radio telescope, the Owens Valley Radio Observatory (OVRO) $40 \mathrm{~m}$ telescope, the $26 \mathrm{~m}$ radio telescope at the University of Michigan Radio Astronomy Observatory (UMRAO), and the 600 meter ring radio telescope RATAN-600. The millimeter interferometer was the Submillimeter Array (SMA). The NRAO Very Long Baseline Array (VLBA) was used for the VLBI observations. For the single-dish instruments and SMA, Mrk 421 is pointlike and unresolved at all observing frequencies. Consequently, the single-dish measurements denote the total flux density of the source integrated over the whole source extension. Details of the observing strategy and data reduction are given by Fuhrmann et al. (2008); Angelakis et al. (2008; F-GAMMA project), Teräsranta et al. (1998; Metsähovi), Aller et al. (1985; UMRAO), Venturi et al. (2001; Medicina and Noto), Kovalev et al. (1999; RATAN-600), and Richards et al. (2011; OVRO).

The VLBA data were obtained at various frequencies $(5,8,15$, 24 , and $43 \mathrm{GHz}$ ) through various programs (BP143, BK150, and Monitoring of Jets in Active galactic nuclei with VLBA Experiments (MOJAVE)). The data were reduced following standard procedures for data reduction and calibration (see, for example, Lister et al. 2009; Sokolovsky et al. 2010, for a description of the MOJAVE and BK150 programs, respectively). Since the VLBA angular resolution is smaller than the radio source extension, measurements were performed for the most compact core region, as well as for the total radio structure at parsec scales. The core is partially resolved by our 15, 24 and $43 \mathrm{GHz}$ observations according to the resolution criterion proposed by Kovalev et al. (2005) and Lobanov (2005). The VLBA core size was determined with two-dimensional Gaussian fits to the measured visibilities. The FWHM size of the core was estimated to be in the range of $0.06-0.12$ mas at the highest observing frequencies, 15, 24 and $43 \mathrm{GHz}$. Both the total and the core radio flux densities from the VLBA data are shown in Figure 8.

\subsubsection{Optical and Near-infrared Instruments}

The coverage at optical frequencies was provided by various telescopes around the globe, and this decreased the sensitivity to weather and technical difficulties and provided good overall coverage of the source, as depicted in Figure 6. Many of the observations were performed within the GASP-WEBT program (e.g., Villata et al. 2008, 2009); this is the case for the data collected by the telescopes at Abastumani, Lulin, Roque de los Muchachos (KVA), St. Petersburg, Talmassons, and Valle d'Aosta observatories ( $R$ band). In addition, the Goddard Robotic Telescope (GRT), the Remote Observatory for Variable Object Research (ROVOR), the New Mexico Skies telescopes, and the Multicolor Imaging Telescopes for Survey and Monstrous Explosions (MITSuME) provided data with various optical filters, while the Guillermo Haro Observatory (OAGH) and the Wyoming Infrared Observatory (WIRO) provided data at near-IR wavelengths. See Table 2 for further details.

All the optical and near-IR instruments used the calibration stars reported in Villata et al. (1998), and the Galactic extinction was corrected with the coefficients given in Schlegel et al. (1998). The flux from the host galaxy (which is significant only below $v \sim 10^{15} \mathrm{~Hz}$ ) was estimated using the flux values at the $R$ band from Nilsson et al. (2007) and the colors reported in Fukugita et al. (1995), and then subtracted from the measured flux.

\subsubsection{Swift/UVOT}

The Swift Ultraviolet and Optical Telescope (UVOT; Roming et al. 2005) data set includes all the observations performed during the time interval MJD 54858-54979, which amounts to 46 single pointing observations that were requested to provide UV coverage during the Mrk 421 multifrequency campaign. The UVOT telescope cycled through each of three ultraviolet passbands (UVW1, UVM2, and UVW2). Photometry was computed using a five-arcsecond source region around Mrk 421 using a custom UVOT pipeline that performs the calibrations presented in Poole et al. (2008). Moreover, the custom pipeline also allows for separate, observation-by-observation corrections for astrometric misalignments (Acciari et al. 2011). A visual inspection was also performed on each of the observations to ensure proper data quality selection and correction. The flux measurements obtained have been corrected for Galactic extinction $E_{B-V}=0.019$ mag (Schlegel et al. 1998) in each spectral band (Fitzpatrick 1999).

\subsubsection{Swift/XRT}

All the Swift X-Ray Telescope (XRT; Burrows et al. 2005) Windowed Timing observations of Mrk 421 carried out from MJD 54858 to 54979 were used for the analysis; this amounts to a total of 46 observations that were performed within this dedicated multi-instrument effort. The XRT data set was first processed with the XRTDAS software package (v.2.5.0) developed at the ASI Science Data Center (ASDC) and distributed by HEASARC within the HEASoft package (v.6.7). Event files were calibrated and cleaned with standard filtering criteria with the xrtpipeline task using the latest calibration files available in the Swift CALDB. The individual XRT event files were then merged together using the XSELECT package and the average spectrum was extracted from the summed event file. Events for the spectral analysis were selected within a circle with a 20 pixel $\left(\sim 47^{\prime \prime}\right)$ radius, which encloses about $95 \%$ of the point-spread function (PSF), centered on the source position. The background was extracted from a nearby circular region with a 40 pixel radius. The source spectrum was binned to ensure a minimum of 20 counts per bin to utilize the $\chi^{2}$ minimization fitting technique. In addition, we needed to apply a small energy offset $(\sim 40 \mathrm{eV})$ to the observed energy spectrum. The origin of this correction is likely to be CCD charge traps generated by radiation and high-energy proton damage (SWIFT-XRT-CALDB-12), which affect mostly the lowest energies (first 1-2 bins) of the spectrum. The ancillary response files were generated with the xrtmkarf task applying corrections for the PSF losses and CCD defects using the cumulative exposure map. The latest response matrices (v.011) available in the Swift CALDB were used.

The XRT average spectrum in the $0.3-10 \mathrm{keV}$ energy band was fitted using the XSPEC package. We adopted a logparabolic model of the form $F(E)=K \times\left(\frac{E}{\mathrm{keV}}\right)^{-\left(\Gamma+\beta \times \log \left(\frac{E}{\mathrm{keV}}\right)\right)}$ (Massaro et al. 2004a, 2004b) with an absorption hydrogenequivalent column density fixed to the Galactic value in the direction of the source, which is $1.61 \times 10^{20} \mathrm{~cm}^{-2}$ (Kalberla et al. 2005). We found that this model provided a good description of the observed spectrum, with the exception of 
the 1.4-2.3 keV energy band where spectral fit residuals were present. These residuals are due to known XRT calibration uncertainties (SWIFT-XRT-CALDB-12 ${ }^{125}$ ) and hence we decided to exclude the $1.4-2.3 \mathrm{keV}$ energy band from the analysis. The resulting spectral fit gave the following parameters: $K=(1.839 \pm 0.002) \times 10^{-1} \mathrm{ph} \mathrm{cm}^{-2} \mathrm{~s}^{-1} \mathrm{keV}^{-1}$, $\Gamma=2.178 \pm 0.002$, and $\beta=0.391 \pm 0.004$. The XRT SED data shown in Figure 8 were corrected for the Galactic absorption and then binned in 16 energy intervals.

\subsubsection{RXTE/PCA}

The Rossi X-Ray Timing Explorer (RXTE; Bradt et al. 1993) satellite performed 59 pointing observations of Mrk 421 during the time interval MJD 54851-54972. These observations amount to a total exposure of $118 \mathrm{ks}$, which was requested through a dedicated Cycle 13 proposal to provide X-ray coverage for this multi-instrument campaign on Mrk 421.

The data analysis was performed using FTOOLS v6.9 and following the procedures and filtering criteria recommended by the RXTE Guest Observer Facility ${ }^{126}$ after 2007 September. The average net count rate from Mrk 421 was about 25 counts $\mathrm{s}^{-1}$ per pcu (in the energy range 3-20 keV) with flux variations typically much smaller than a factor of two. Consequently, the observations were filtered following the conservative procedures for faint sources: Earth elevation angle greater than $10^{\circ}$, pointing offset less than 0.02 , time since the peak of the last SAA (South Atlantic Anomaly) passage greater than 30 minutes, and electron contamination less than 0.1 . For further details on the analysis of faint sources with RXTE, see the online Cook Book. ${ }^{127}$ In the data analysis, in order to increase the quality of the signal, only the first xenon layer of PCU2 was used. We used the package pcabackest to model the background and the package saextrct to produce spectra for the source and background files and the script ${ }^{128}$ pcarsp to produce the response matrix.

The Proportional Counter Array (PCA) average spectrum in the 3-32 keV energy band was fitted using the XSPEC package using a PL function with an exponential cutoff (cutoffpl) with a non-variable neutral hydrogen column density $N_{\mathrm{H}}$ fixed to the Galactic value in the direction of the source $\left(1.61 \times 10^{20} \mathrm{~cm}^{-2}\right.$; Kalberla et al. 2005). However, since the PCA bandpass starts at $3 \mathrm{keV}$, the value for $N_{\mathrm{H}}$ used does not significantly affect our results. The resulting spectral fit provided a good representation of the data for the following parameters: normalization parameter $K=(2.77 \pm 0.03) \times 10^{-1} \mathrm{ph} \mathrm{cm}^{-2} \mathrm{~s}^{-1} \mathrm{keV}^{-1}$, photon index $\Gamma=2.413 \pm 0.015$, and cutoff energy $E_{\exp }=22.9 \pm 1.3 \mathrm{keV}$. The obtained 23 energy bins' PCA average spectrum is shown in Figure 8.

\subsubsection{Swift/BAT}

The Swift/BAT (Barthelmy et al. 2005) analysis results presented in this paper were derived with all the available data during the time interval MJD 54850-54983. The spectrum was extracted following the recipes presented in Ajello et al. (2008, $2009 \mathrm{~b}$ ). This spectrum is constructed by weight averaging the source spectra extracted over short exposures (e.g., $300 \mathrm{~s}$ ) and it is representative of the averaged source emission over the time

\footnotetext{
125 http://heasarc.gsfc.nasa.gov/docs/heasarc/caldb/swift/docs/xrt/ SWIFT-XRT-CALDB-09_v12.pdf

${ }^{126} \mathrm{http}$ ://www.universe.nasa.gov/xrays/programs/rxte/pca/doc/bkg/ bkg-2007-saa/

127 http://heasarc.gsfc.nasa.gov/docs/xte/recipes/cook_book.html

${ }^{128}$ The CALDB files are located at http://heasarc.gsfc.nasa.gov/FTP/caldb.
}

range spanned by the observations. These spectra are accurate to the mCrab level and the reader is referred to Ajello et al. (2009a) for more details. The Swift/BAT spectrum in the $15-200 \mathrm{keV}$ energy range is consistent with a PL function with normalization parameter $K=0.46 \pm 0.27 \mathrm{ph} \mathrm{cm}^{-2} \mathrm{~s}^{-1} \mathrm{keV}^{-1}$ and photon index $\Gamma=3.0 \pm 0.3$. The last two flux points are within one standard deviation from the above-mentioned PL function, and hence the apparent upturn given by these last two data points in the spectrum is not significant.

\subsubsection{MAGIC}

MAGIC is a system of two $17 \mathrm{~m}$ diameter IACTs for VHE $\gamma$-ray astronomy located on the Canary Island of La Palma, at an altitude of $2200 \mathrm{~m}$ above sea level. At the time of the observation, MAGIC-II, the new second telescope of the current array system, was still in its commissioning phase so that Mrk 421 was observed in stand-alone mode by MAGIC-I, which has been in scientific operation since 2004 (Albert et al. 2008). The MAGIC observations were performed in the socalled wobble mode (Daum 1997). In order to have a low energy threshold, only observations at zenith angles less than $35^{\circ}$ were used in this analysis. The bad weather and a shutdown for a scheduled hardware system upgrade during the period MJD 54948-54960 (April 27-May 13) significantly reduced the amount of time that had initially been scheduled for this campaign. The data were analyzed following the prescription given by Albert et al. (2008) and Aliu et al. (2009). The data surviving the quality cuts amounted to a total of $27.7 \mathrm{hr}$. The preliminary reconstructed photon fluxes for the individual observations gave an average flux of about $50 \%$ that of the Crab Nebula, with relatively mild (typically less than a factor of two) flux variations. The derived spectrum was unfolded to correct for the effects of the limited energy resolution of the detector and possible bias (Albert et al. 2007c). The resulting spectrum was fit satisfactorily with a single logparabola function: $F(E)=K \times(E / 0.3 \mathrm{TeV})^{-(\Gamma+\beta \cdot \log (E / 0.3 \mathrm{TeV}))}$. The resulting spectral fit gave the following parameters: $K=$ $(6.50 \pm 0.13) \times 10^{-10} \mathrm{ph} \mathrm{cm}^{-2} \mathrm{~s}^{-1} \mathrm{erg}^{-1}, \Gamma=2.48 \pm 0.03$, and $\beta=0.33 \pm 0.06$, with $\chi^{2} / N D F=11 / 6$. A fit with a simple PL function gives $\chi^{2} / N D F=47 / 7$, which confirmed the existence of curvature in the VHE spectrum.

\subsection{Fermi-LAT Spectra during the Campaign}

The Mrk 421 spectrum measured by Fermi-LAT during the period covered by the multifrequency campaign is shown in panel (b) of Figure 7. The spectrum can be described with a single PL function with photon index $1.75 \pm 0.03$ and photon flux $F(>0.3 \mathrm{GeV})=(6.1 \pm 0.3) \times 10^{-8} \mathrm{ph} \mathrm{cm}^{-2} \mathrm{~s}^{-1}$, which is somewhat lower than the average spectrum over the first 1.5 years of Fermi-LAT operation (see Figure 5).

For comparison purposes, we also computed the spectra for the time periods before and after the multifrequency campaign (the time intervals MJD 54683-54850 and MJD 54983-55248, respectively). These two spectra are shown in panels (a) and (c) of Figure 7. The two spectra can be described very satisfactorily with single PL functions of photon indices $1.79 \pm 0.03$ and $1.78 \pm 0.02$ and photon fluxes $F(>0.3 \mathrm{GeV})=(7.1 \pm 0.3) \times 10^{-8} \mathrm{ph} \mathrm{cm}^{-2} \mathrm{~s}^{-1}$ and $F(>0.3 \mathrm{GeV})=(7.9 \pm 0.2) \times 10^{-8} \mathrm{ph} \mathrm{cm}^{-2} \mathrm{~s}^{-1}$. Therefore, during the multifrequency campaign, Mrk 421 showed a spectral shape that is compatible with the periods before and after the campaign, and a photon flux which is about $20 \%$ lower than before the campaign and $30 \%$ lower than after the campaign. 

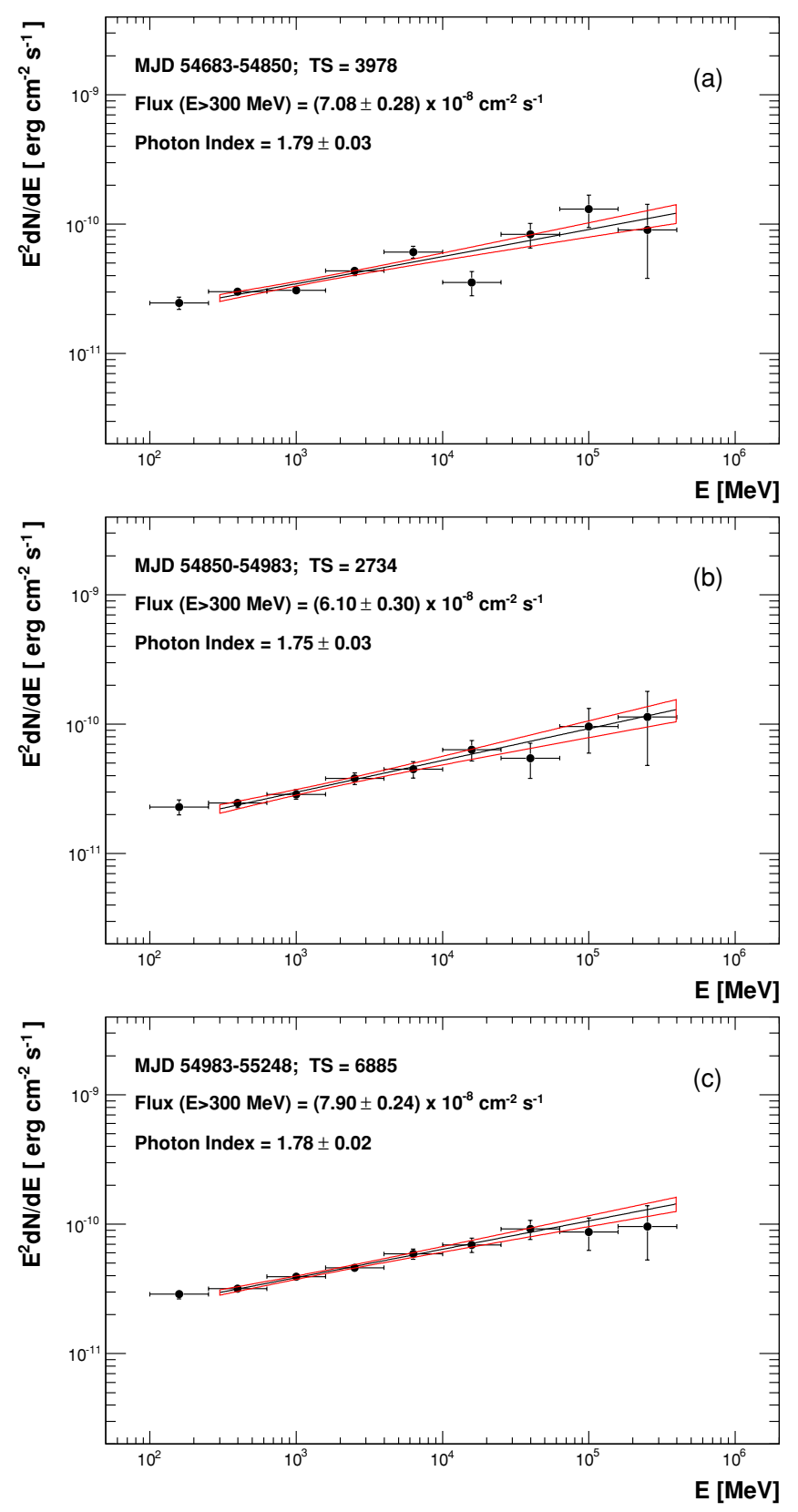

Figure 7. Fermi spectra of Mrk 421 for several time intervals of interest. Panel (a) shows the spectrum for the time period before the multifrequency campaign (MJD 54683-54850), panel (b) for the time interval corresponding to the multifrequency campaign (MJD 54850-54983), and panel (c) for the period after the campaign (MJD 54983-55248). In all panels, the black line depicts the result of the unbinned likelihood PL fit and the red contours denote the $68 \%$ uncertainty of the PL fit. The legend reports the results from the unbinned likelihood PL fit in the energy range $0.3-400 \mathrm{GeV}$.

(A color version of this figure is available in the online journal.)

\subsection{The Average Broadband SED during the Multifrequency Campaign}

The average SED of Mrk 421 resulting from our 4.5 month long multifrequency campaign is shown in Figure 8. This is the most complete SED ever collected for Mrk 421 or for any other BL Lac object (although an SED of nearly similar quality was reported in Abdo et al. 2011b for Mrk 501). At the highest energies, the combination of Fermi-LAT and MAGIC allows us to measure, for the first time, the high energy bump without any gap; both the rising and falling segments of the com- ponents are precisely determined by the data. The low energy bump is also measured very well: Swift/BAT and RXTE/PCA describe its falling part, Swift/XRT describes the peak, and the Swift/UV and the various optical and IR observations describe the rising part. The rising tail of this peak was also measured with various radio instruments. Especially important are the observations from SMA at $225 \mathrm{GHz}$, which help connect the bottom (radio) to the peak (optical/X-rays) of the synchrotron bump (in the $v F_{v}$ representation). The flux measurements by VLBA, especially the ones corresponding to the core, provide us with the radio flux density from a region that is presumably not much larger than the blazar emission region. Therefore, the radio flux densities from interferometric observations (from the VLBA core) are expected to be close upper limits to the radio continuum of the blazar emission component. On the other hand, the low frequency radio observations performed with single dish instruments have a relatively large contamination from the nonblazar emission and are probably considerably above the energy flux from the blazar emission region. The only spectral intervals lacking observations are $1 \mathrm{meV}-0.4 \mathrm{eV}$, and $200 \mathrm{keV}-100 \mathrm{MeV}$, where the sensitivity of the current instruments is insufficient to detect Mrk 421. We note, however, that the detailed GeV coverage together with our broadband, one-zone SSC modeling strongly constrains the expected emission in the difficult-toaccess $1 \mathrm{meV}-0.4 \mathrm{eV}$ bandpass.

During this campaign, Mrk 421 showed low activity and relatively small flux variations at all frequencies (Paneque 2009). At VHE ( $>100 \mathrm{GeV})$, the measured flux is half the flux from the Crab Nebula, which is among the lowest fluxes recorded by MAGIC for this source (Albert et al. 2007a; Aleksić et al. 2010). At X-rays, the fluxes observed during this campaign are about 15 $\mathrm{mCrab}$, which is about three times higher than the lowest fluxes measured by RXTE/ASM since 1996. Therefore, because of the low flux, low (multifrequency) variability, and the large density of observations, the collected data during this campaign can be considered an excellent proxy for the low/quiescent state SED of Mrk 421. It is worth stressing that the good agreement in the overlapping energies of the various instruments (which had somewhat different time coverages during the campaign) supports this hypothesis.

\section{SED MODELING}

We turn now to modeling the multifrequency data set collected during the 4.5 month campaign in the context of homogeneous hadronic and leptonic models. The models discussed below assume emission mainly from a single, spherical, and homogeneous region of the jet. This is a good approximation to model flaring events with observed correlated variability (where the dynamical timescale does not exceed the flaring timescale significantly), although it is an oversimplification for quiescent states, where the measured blazar emission might be produced by the radiation from different zones characterized by different values of the relevant parameters. There are several models in the literature along those lines (e.g., Ghisellini et al. 2005; Katarzyński et al. 2008; Graff et al. 2008; Giannios et al. 2009) but at the cost of introducing more free parameters that are, consequently, less well constrained and more difficult to compare between models. This is particularly problematic if a "limited" data set (in time and energy coverage) is employed in the modeling, although it could work well if the amount of multifrequency data is extensive enough to substantially constrain the parameter space. In this work, we adopted the one-zone homogeneous models for their simplicity as well as for being able to compare 


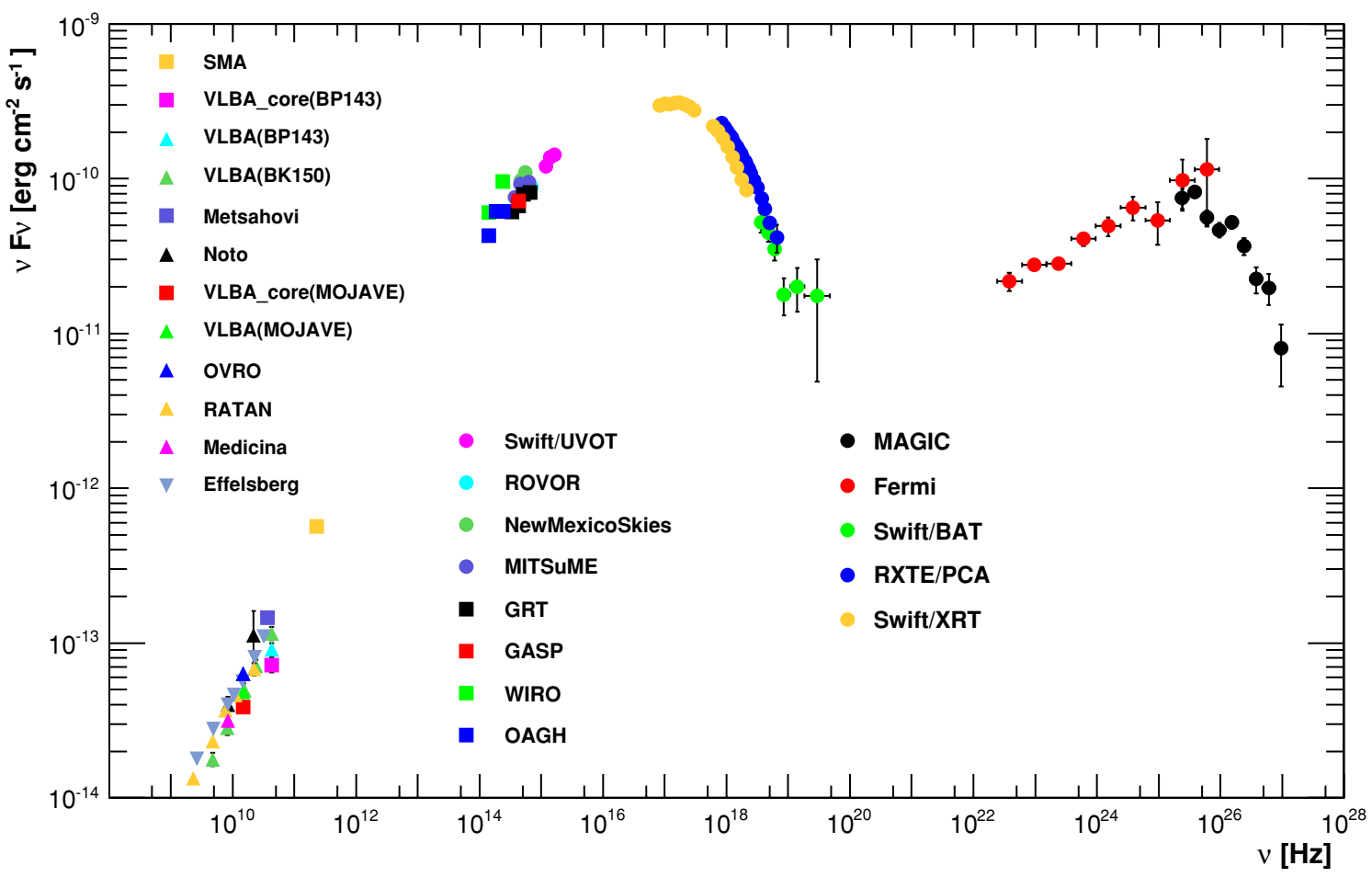

Figure 8. Spectral energy distribution of Mrk 421 averaged over all the observations taken during the multifrequency campaign from 2009 January 19 (MJD 54850 ) to 2009 June 1 (MJD 54983). The legend reports the correspondence between the instruments and the measured fluxes. The host galaxy has been subtracted, and the optical/X-ray data were corrected for the Galactic extinction. The TeV data from MAGIC were corrected for the absorption in the EBL using the prescription given in Franceschini et al. (2008).

with previous works. The one-zone homogeneous models are the most widely used models to describe the SED of high-peaked BL Lac objects. Furthermore, although the modeled SED is averaged over 4.5 months of observations, the very low observed multifrequency variability during this campaign, and in particular the lack of strong $\mathrm{keV}$ and $\mathrm{GeV}$ variability (see Figures 1 and 2 ) in these timescales, suggests that the presented data are a good representation of the average broadband emission of Mrk 421 on timescales of a few days. We therefore feel confident that the physical parameters required by our modeling to reproduce the average 4.5 month SED are a good representation of the physical conditions at the emission region down to timescales of a few days, which is comparable to the dynamical timescale derived from the models we discuss. The implications (and caveats) of the modeling results are discussed in Section 7.

Mrk 421 is at a relatively low redshift $(z=0.031)$, yet the attenuation of its VHE MAGIC spectrum by the extragalactic background light (EBL) is non-negligible for all models and hence needs to be accounted for using a parameterization for the EBL density. The EBL absorption at $4 \mathrm{TeV}$, the highest energy bin of the MAGIC data (absorption will be less at lower energies), varies according to the model used from $e^{-\tau_{\gamma \gamma}}=0.29$ for the "Fast Evolution" model of Stecker et al. (2006) to $e^{-\tau_{\gamma \gamma}}=0.58$ for the models of Franceschini et al. (2008) and Gilmore et al. (2009), with most models giving $e^{-\tau_{\gamma \gamma}} \sim 0.5-0.6$, including the model of Finke et al. (2010) and the "best fit" model of Kneiske et al. (2004). We have de-absorbed the TeV data from MAGIC with the Franceschini et al. (2008) model, although most other models give comparable results.

\subsection{Hadronic Model}

If relativistic protons are present in the jet of Mrk 421, hadronic interactions, if above the interaction threshold, must

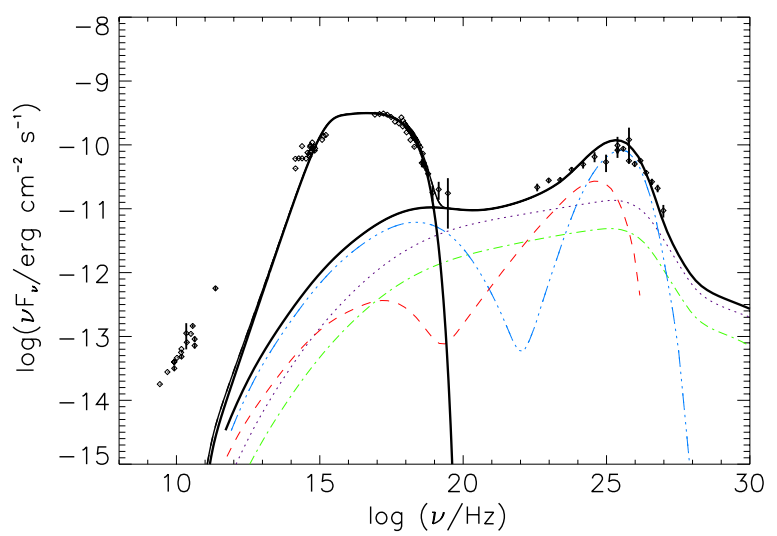

Figure 9. Hadronic model fit components: $\pi^{0}$-cascade (black dotted line), $\pi^{ \pm}$ cascade (green dash-dotted line), $\mu$-synchrotron and cascade (blue triple-dotdashed line), and proton synchrotron and cascade (red dashed line). The black thick solid line is the sum of all emission components (which also includes the synchrotron emission of the primary electrons at optical/X-ray frequencies). The resulting model parameters are reported in Table 3 .

be considered for modeling the source emission. For the present modeling, we use the hadronic Synchrotron-Proton Blazar (SPB) model of Mücke et al. (2001, 2003). Here, the relativistic electrons $(e)$ injected in the strongly magnetized (with homogeneous magnetic field with strength $B$ ) blob lose energy predominantly through synchrotron emission. The resulting synchrotron radiation of the primary $e$ component dominates the low energy bump of the blazar SED, and serves as target photon field for interactions with the instantaneously injected relativistic protons (with index $\alpha_{p}=\alpha_{e}$ ) and pair (synchrotron-supported) cascading.

Figures 9 and 10 show a satisfactory (single zone) SPB model representation of the data from Mrk 421 collected during the 


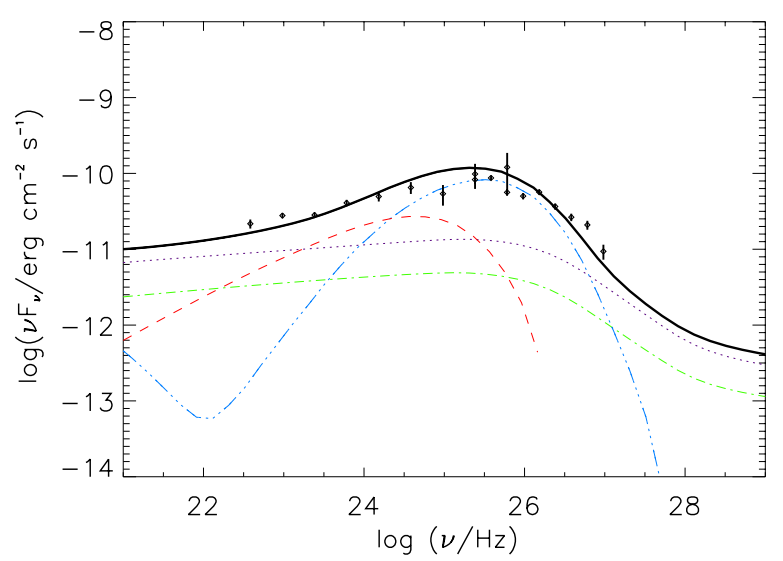

Figure 10. Expanded view of the high energy bump of the SED data and model presented in Figure 9.

Table 3

Parameter Values from the SPB Model Fit to the SED from Mrk 421 Shown in Figure 9

\begin{tabular}{|c|c|c|}
\hline Parameter & Symbol & Value \\
\hline Doppler factor & $\delta$ & 12 \\
\hline Magnetic field (G) & $B$ & 50 \\
\hline Comoving blob radius $(\mathrm{cm})$ & $R$ & $4 \times 10^{14}$ \\
\hline Power-law index of the injected electron distribution ${ }^{a}$ & $\alpha_{e}$ & 1.9 \\
\hline Power-law index of the injected proton distribution ${ }^{\mathrm{a}}$ & $\alpha_{p}$ & 1.9 \\
\hline Minimum electron Lorentz factor & $\gamma_{e, \min }$ & $7 \times 10^{2}$ \\
\hline Maximum electron Lorentz factor & $\gamma_{e, \max }$ & $4 \times 10^{4}$ \\
\hline Minimum proton Lorentz factor ${ }^{\mathrm{b}}$ & $\gamma_{p, \min }$ & 1 \\
\hline Maximum proton Lorentz factor & $\gamma_{p, \max }$ & $2.3 \times 10^{9}$ \\
\hline Energy density in protons $\left(\mathrm{erg} \mathrm{cm}^{-3}\right)$ & $u_{p}^{\prime}$ & 510 \\
\hline Ratio of number of electrons with respect to protons & $e / p$ & 90 \\
\hline Jet power $\left(\mathrm{erg} \mathrm{s}^{-1}\right)$ & $P_{\text {jet }}$ & $4.5 \times 10^{44}$ \\
\hline
\end{tabular}

Notes.

a The model assumes $\alpha_{e}=\alpha_{p}$, hence only one free parameter.

$\mathrm{b}$ The parameter $\gamma_{p \text {,min }}$ was fixed to the lowest possible value, 1 , and hence this is actually not a free parameter.

campaign. The corresponding parameter values are reported in Table 3. In order to fit the optical data, the lowest energy of the injected electrons is required to be maintained as $\gamma_{e, \min } \approx 700$ through the steady state. This requires a continuous electron injection rate density of at least $\gtrsim 1.4 \mathrm{~cm}^{-3} \mathrm{~s}^{-1}$ to balance the synchrotron losses at that energy, and is a factor of $\sim 100$ larger than the proton injection rate. The radio fluxes predicted by the model are significantly below the observed $8-230 \mathrm{GHz}$ radio fluxes. This is related to the model being designed to follow the evolution of the jet emission during $\gamma$-ray production where radiative cooling dominates over adiabatic cooling. Here, the emission region is optically thick up to $\sim 100 \mathrm{GHz}$ frequencies, and the synchrotron cooling break $\left(\gamma_{e} \sim 10\right)$ would be below the synchrotron-self-absorption turnover. The introduction of additional, poorly constrained components would be necessary to account for the subsequent evolution of the jet through the expansion phase where the synchrotron radiation becomes gradually optically thin at centimeter wavelengths. This is omitted in the following modeling.

The measured spectra in the $\gamma$-ray band $(>1 \mathrm{GeV})$ is dominated by synchrotron radiation from short-lived muons (produced during photomeson production) as well as proton synchrotron radiation, with significant overall reprocessing, while below this energy the $\pi$-cascade dominates. The interplay between muon and proton synchrotron radiation together with appreciable cascade synchrotron radiation initiated by the pairs and high energy photons from photomeson production, is responsible for the observed $\mathrm{MeV}-\mathrm{GeV}$ flux. The $\mathrm{TeV}$ emission is dominated by the high energy photons from the muon synchrotron component. The source intrinsic model SED predicts $>10 \mathrm{TeV}$ emission on a level of two to three orders of magnitude below the sub-TeV flux, which will be further weakened by $\gamma$-ray absorption by the EBL.

The overall required particle and field energy density are within a factor of five of equipartition, and a total jet power (as measured in the galaxy rest frame) of $4 \times 10^{44} \mathrm{erg} \mathrm{s}^{-1}$ in agreement with expectations for a weakly accreting disk of a BL Lac object (see Cao 2003).

Alternative model fits are possible if the injected electron and proton components do not have the same PL index. This "relaxation" of the model would add one extra parameter and so would allow for improvement in the data-model agreement, especially around the synchrotron peak and the high energy bump. It would also allow a larger tolerance on the size region $\mathrm{R}$, which is considered to be small in the SPB model fit presented here.

\subsection{Leptonic Model}

The simplest leptonic model typically used to describe the emission from BL Lac objects is the one-zone SSC model. Within this framework, the radio through X-ray emission is produced by synchrotron radiation from electrons in a homogeneous, randomly oriented magnetic field $(B)$ and the $\gamma$-rays are produced by inverse Compton scattering of the synchrotron photons by the same electrons which produce them. For this purpose, we use the one-zone SSC code described in Finke et al. (2008). The electron distribution from one-zone SSC models is typically parameterized with one or two PL functions (that is, zero breaks or one break) within an electron Lorentz factor range defined by $\gamma_{\min }$ and $\gamma_{\max }$ (where the electron energy is $\gamma m_{e} c^{2}$ ). We use the same approach in this work. However, we find that, in order to properly describe the shape of the measured broadband SED during the 4.5 month long campaign, the model requires an electron distribution parameterized with three PL functions (and hence two breaks). In other words, we must add two extra free parameters to the model: the second break at $\gamma_{\text {brk,2 }}$ and the index of the third PL function $p_{3}$. Note that a second break was also needed to describe the SED of Mrk 501 in the context of the synchrotron/SSC model (Abdo et al. 2011b). An alternative possibility might be to use an electron distribution parameterized with a curved function such as that resulting from episodic particle acceleration (Perlman et al. 2005) or the log-parabolic function used in Tramacere et al. (2009). However, we note that such a parameterization might have problems describing the highest $\mathrm{X}$-ray energies, where the current SED data (RXTE/PCA and Swift/BAT) do not show a large spectrum curvature.

Even though the very complete SED constrains the shape of the electron distribution quite well, there is still some degeneracy in the range of allowed values for the general source parameters $R$ (comoving blob radius), $B$, and $\delta$ (doppler factor). For a given break in the measured low energy (synchrotron) bump, the break in the electron distribution $\gamma_{\text {brk }}$ scales as $1 / \sqrt{B \delta}$. In order to minimize the range of possible parameters, we note that the emitting region radius is constrained by the variability time, $t_{v}$, 


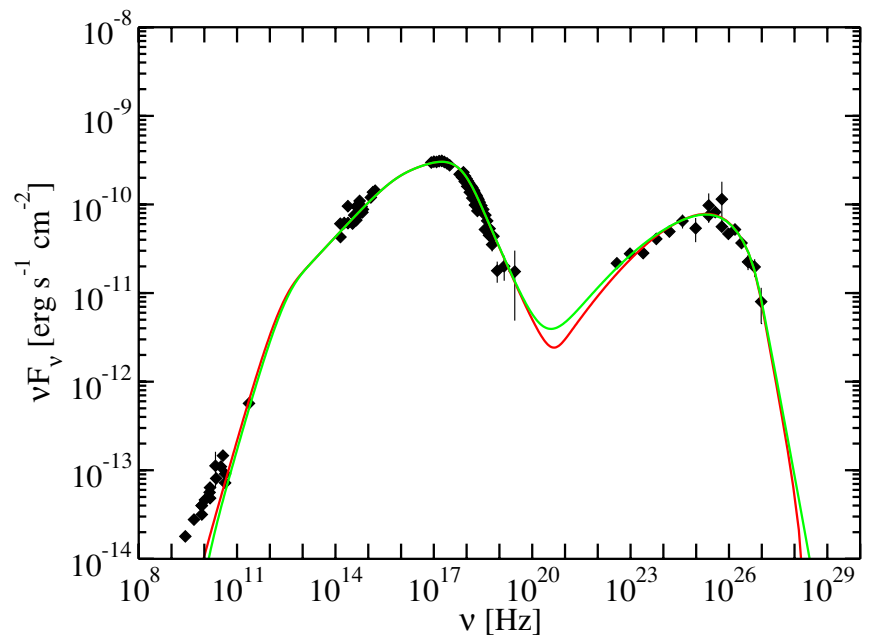

Figure 11. SED of Mrk 421 with two one-zone SSC model fits obtained with different minimum variability timescales: $t_{\mathrm{var}}=1$ day (red curve) and $t_{\mathrm{var}}=1$ hr (green curve). The parameter values are reported in Table 4. See the text for further details.

Table 4

Parameter Values from the One-zone SSC Model Fits to the SED from Mrk 421 Shown in Figure 11

\begin{tabular}{|c|c|c|c|}
\hline Parameter & Symbol & Red Curve & Green Curve \\
\hline Variability timescale $(\mathrm{s})^{\mathrm{a}}$ & $t_{v, \min }$ & $8.64 \times 10^{4}$ & $3.6 \times 10^{3}$ \\
\hline Doppler factor & $\delta$ & 21 & 50 \\
\hline Magnetic field (G) & $B$ & $3.8 \times 10^{-2}$ & $8.2 \times 10^{-2}$ \\
\hline Comoving blob radius $(\mathrm{cm})$ & $R$ & $5.2 \times 10^{16}$ & $5.3 \times 10^{15}$ \\
\hline Low-energy electron spectral index & $p_{1}$ & 2.2 & 2.2 \\
\hline Medium-energy electron spectral index & $p_{2}$ & 2.7 & 2.7 \\
\hline High-energy electron spectral index & $p_{3}$ & 4.7 & 4.7 \\
\hline Minimum electron Lorentz factor & $\gamma_{\min }$ & $8.0 \times 10^{2}$ & $4 \times 10^{2}$ \\
\hline Break1 electron Lorentz factor & $\gamma_{\text {brk1 }}$ & $5.0 \times 10^{4}$ & $2.2 \times 10^{4}$ \\
\hline Break2 electron Lorentz factor & $\gamma_{\text {brk2 }}$ & $3.9 \times 10^{5}$ & $1.7 \times 10^{5}$ \\
\hline Maximum electron Lorentz factor & $\gamma_{\max }$ & $1.0 \times 10^{8}$ & $1.0 \times 10^{8}$ \\
\hline Jet power in magnetic field $\left(\mathrm{erg} \mathrm{s}^{-1}\right)^{\mathrm{b}} \mathrm{x}$ & $P_{j, B}$ & $1.3 \times 10^{43}$ & $3.6 \times 10^{42}$ \\
\hline Jet power in electrons $\left(\mathrm{erg} \mathrm{s}^{-1}\right)$ & $P_{j, e}$ & $1.3 \times 10^{44}$ & $1.0 \times 10^{44}$ \\
\hline Jet power in photons $\left(\operatorname{erg~s}^{-1}\right)^{b}$ & $P_{j, p h}$ & $6.3 \times 10^{42}$ & $1.1 \times 10^{42}$ \\
\hline
\end{tabular}

Notes.

a The variability timescale was not derived from the model fit, but rather used as an input (constrain) to the model. See the text for further details.

b The quantities $P_{j, B}$ and $P_{j, p h}$ are derived quantities; only $P_{j, e}$ is a free parameter in the model.

so that

$$
R=\frac{\delta c t_{v, \min }}{1+z} \leqslant \frac{\delta c t_{v}}{1+z} .
$$

During the observing campaign, Mrk 421 was in a rather low activity state, with multifrequency flux variations occurring on timescales larger than one day (Paneque 2009), so we used $t_{v \text {, min }}=1$ day in our modeling. In addition, given that this only gives an upper limit on the size scale, and the history of fast variability detected for this object (e.g., Gaidos et al. 1996; Giebels et al. 2007), we also performed the SED model using $t_{v \text {, min }}=1 \mathrm{hr}$. The resulting SED models obtained with these two variability timescales are shown in Figure 11, with the parameter values reported in Table 4 . The blob radii are large enough in these models that synchrotron self-absorption (SSA) is not important; for the $t_{v, \min }=1 \mathrm{hr}$ model, $v_{\mathrm{SSA}}=3 \times 10^{10} \mathrm{~Hz}$, at which frequency a break is barely visible in Figure 11. It is worth stressing the good agreement between the model and the data: the model describes very satisfactorily the entire measured broadband SED. The model goes through the SMA $(225 \mathrm{GHz})$ data point, as well as through the VLBA (43 GHz) data point for the partially resolved radio core. The size of the VLBA core of the 2009 data from Mrk 421 at $15 \mathrm{GHz}$ and $43 \mathrm{GHz}$ is $\simeq 0.06-0.12$ mas (as reported in Section 5.1.1) or using the conversion scale $0.61 \mathrm{pc} \mathrm{mas}^{-1} \simeq 1-2 \times 10^{17} \mathrm{~cm}$. The VLBA size estimation is the FWHM of a Gaussian representing the brightness distribution of the blob, which could be approximated as 0.9 times the radius of a corresponding spherical blob (Marscher 1983). That implies that the size of the VLBA core is comparable (a factor of about two to four times larger) than that of the model blob for $t_{\mathrm{var}}=1$ day $\left(\sim 5 \times 10^{16} \mathrm{~cm}\right)$. Therefore, it is reasonable to consider that the radio flux density from the VLBA core is indeed dominated by the radio flux density of the blazar emission. The other radio observations are single dish measurements and hence integrate over a region that is orders of magnitude larger than the blazar emission. Consequently, we treat them as upper limits for the model.

The powers of the different jet components derived from the model fits (assuming $\Gamma=\delta$ ) are also reported in Table 4. Estimates for the mass of the supermassive black hole in Mrk 421 range from $2 \times 10^{8} M_{\odot}$ to $9 \times 10^{8} M_{\odot}$ (Barth et al. 2003; Wu et al. 2002), and hence the Eddington luminosity should be between $2.6 \times 10^{46}$ and $1.2 \times 10^{47} \mathrm{erg} \mathrm{s}^{-1}$, that is, well above the jet luminosity.

It is important to note that the parameters resulting from the modeling of our broadband SED differ somewhat from the parameters obtained for this source of previous works (Krawczynski et al. 2001; Błażejowski et al. 2005; Revillot et al. 2006; Albert et al. 2007b; Giebels et al. 2007; Fossati et al. 2008; Finke et al. 2008; Horan et al. 2009; Acciari et al. 2009). One difference, as already noted, is that an extra break is required. This could be a feature of Mrk 421 in all states, but we only now have the simultaneous high quality spectral coverage to identify it. For the model with $t_{\mathrm{var}}=1$ day (which is the time variability observed during the multifrequency campaign), additional differences with previous models are in $R$, which is an order of magnitude larger, and $B$, which is an order of magnitude smaller. This mostly results from the longer variability time in this low state. Note that using a shorter variability $\left(t_{\mathrm{var}}=1 \mathrm{hr}\right.$; green curve) gives a smaller $R$ and bigger $B$ than most models of this source.

Another difference in our one-zone SSC model with respect to previous works relates to the parameter $\gamma_{\min }$. This parameter has typically not been well constrained because the single-dish radio data can only be used as upper limits for the radio flux from the blazar emission. This means that the obtained value for $\gamma_{\text {min }}$ (for a given set of other parameters $R, B$, and $\delta$ ) can only be taken as a lower limit: a higher value of $\gamma_{\min }$ is usually possible. In our modeling we use simultaneous Fermi-LAT data as well as SMA and VLBA radio data, which we assume are dominated by the blazar emission. We note that the size of the emission from our SED model fit (when using $t_{\mathrm{var}} \sim 1$ day) is comparable to the partially resolved VLBA radio core and hence we think this assumption is reasonable. The requirement that the model SED fit goes through those radio points further constrains the model, and in particular the parameter $\gamma_{\min }$ : a decrease in the value of $\gamma_{\min }$ would overpredict the radio data, while an increase of $\gamma_{\text {min }}$ would underpredict the SMA and VLBA core radio data, as well as the Fermi-LAT spectrum below $1 \mathrm{GeV}$ if the increase in $\gamma_{\min }$ would be large. We explored model fits with different $\gamma_{\text {min }}$ and $p_{1}$, and found that, for the SSC model fit with $t_{\mathrm{var}}=1$ day 


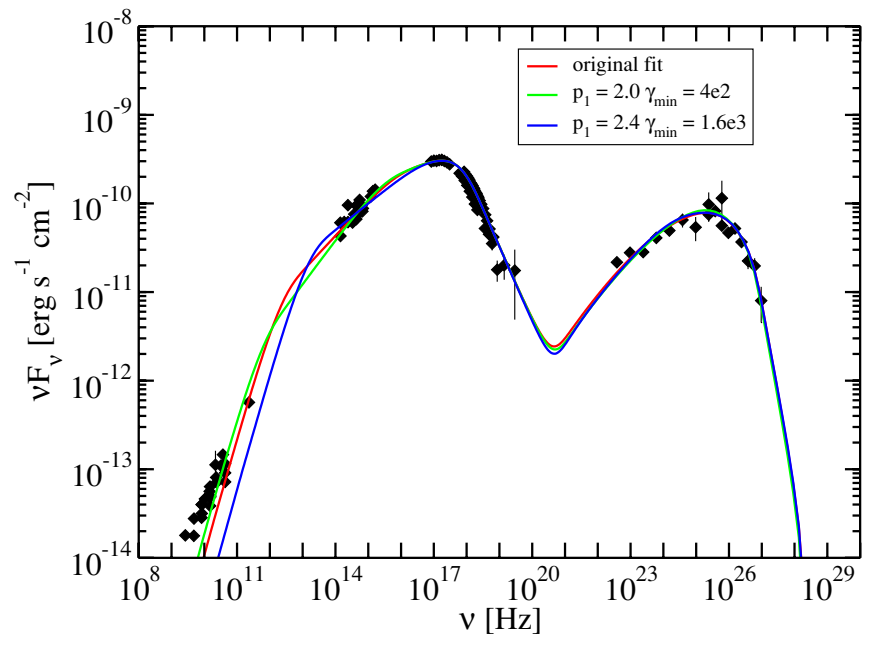

Figure 12. SSC model fit of the SED from Mrk 421 presented in Figure 11 (for $t_{\mathrm{var}} \sim 1$ day), with variations by a factor of two of the parameter $\gamma_{\mathrm{min}}$, together with adjustments in the parameter $p_{1}$ in order to match the experimental data. See the text for further details.

(red curve in Figure 11), $\gamma_{\min }$ is well constrained within a factor of two to the value of $8 \times 10^{2}$ (see Figure 12). In the case of the SSC model with $t_{\mathrm{var}}=1 \mathrm{hr}$ (green curve in Figure 11), if we make the same assumption that the SMA and VLBA core emission is dominated by the blazer emission, ${ }^{129} \gamma_{\min }$ can be from $2 \times 10^{2}$ up to $10^{3}$, and still provide a good match to the SMA/VLBA/optical data and the Fermi-LAT spectrum. In any case, for any variability timescale, the electron distribution does not extend down to $\gamma_{\text {min }} \sim 1$ to a few, and is constrained within a factor of two. This is particularly relevant because, for PL distributions with index $p>2$, the jet power carried by the electrons is dominated by the low energy electrons. Therefore, the tight constraints on $\gamma_{\text {min }}$ translate into tight constraints on the jet power carried by the electrons. For instance, in the case of the model with $t_{\mathrm{var}}=1 \mathrm{hr}$, using $\gamma_{\min }=10^{3}$ (instead of $\gamma_{\text {min }}=4 \times 10^{2}$ ) would reduce the jet power carried by electrons from $P_{j, e} \approx 10^{44} \mathrm{erg} \mathrm{s}^{-1}$ down to $P_{j, e} \approx 8 \times 10^{43} \mathrm{erg} \mathrm{s}^{-1}$.

Another parameter where the results presented here differ from previous results in the literature is the first PL index $p_{1}$. This parameter is dominated by the optical and UV data points connecting with the Swift/XRT, as well as by the necessity of matching the model with the Fermi-LAT GeV data. Note that our model fit also goes over the SMA and VLBA (partially resolved) core fluxes. Again, since these constraints did not exist (or were not used) in the past, most of the one-zone SSC model results (for Mrk 421) in the literature report a $p_{1}$ value that differs from the one reported in this work. We note, however, that the values for the parameters $p_{2}$ and $p_{3}$ from our model fits, which are constrained mostly by the $\mathrm{X}$-ray/TeV data, are actually quite similar to the parameters $p_{1}$ and $p_{2}$ from the previous one-zone SSC model fits to Mrk 421 data.

\section{DISCUSSION}

In this section of the paper, we discuss the implications of the experimental and SED modeling results presented in the previous sections. As explained at the beginning of Section 6,

\footnotetext{
${ }^{129}$ In the case of $t_{\mathrm{var}} \sim 1 \mathrm{hr}$, the size of the emission region derived from the SSC model is one order of magnitude smaller than the size of the VLBA core and hence the assumption used is somewhat less valid than for the model with $t_{\mathrm{var}} \sim 1$ day.
}

for simplicity and for the sake of comparison with previously published results, we modeled the SED with scenarios based on one-zone homogeneous emitting regions, which are commonly used to parameterize the broadband emission in blazars. We note that this is a simplification of the problem; the emission in blazar jets could be produced in an inhomogeneous or stratified region, as well as in $N$ independent regions. An alternative and quite realistic scenario could be a standing shock where particle acceleration takes place and radiation is being produced as the jet flow or superluminal knots cross it (e.g., Komissarov \& Falle 1997; Marscher et al. 2008). The Lorentz factor of the plasma, as it flows through the standing (and by necessity oblique) shock, is the Lorentz factor (and through setting the angle, the Doppler factor) of the model. We note, however, that, as discussed in Sikora et al. (1997), the steady-state emission could also be parameterized by $N$ moving blobs that only radiate when passing through the standing shock. If at any given moment only one of these blobs were visible at the observer frame, the one-zone homogeneous model could be a plausible approximation of the standing-shock scenario.

In any case, the important thing is that, in the proposed physical scenario, the stability timescale of the particle accelerating shock front is not connected to the much shorter cooling times that give rise to spectral features. For as long as the injection of particles in the blob and the dynamics of the blob remain unchanged, the SED, along with the breaks due to radiative cooling and due to the value of $\gamma_{\text {min }}$ where Fermi acceleration presumably picks up, will remain unchanged. The lack of (substantial) multifrequency variability observed during this campaign suggests that this is the case, and hence that the 4.5 month averaged SED is also representative of the broadband emission of SED during much shorter periods of time that are comparable to the dynamical timescales derived from the models.

\subsection{What are the Spectral Breaks Telling Us?}

In our homogenous leptonic model, we reproduce the location of the $v f_{v}$ peaks by fitting the Lorentz factors $\gamma_{\text {brk,1 }}$ and $\gamma_{\text {brk,2 }}$ (as well as the values of $B$ and $\delta$ ) where the electron energy distribution breaks. There is, however, a Lorentz factor where one typically (in blazar modeling) expects a break in the electron energy distribution (EED), and this is the Lorentz factor $\gamma_{c}=3 \pi m_{e} c^{2} /\left(\sigma_{\tau} B^{2} R\right)$ where the escape time from the source equals the radiative (synchrotron) cooling time. The fact that the values of the second break, $\gamma_{\text {brk,2 }}$, fit by our leptonic models $\left(\gamma_{\text {brk, } 2}=3.9 \times 10^{5}, 1.7 \times 10^{5}\right)$ are similar to the Lorentz factors $\left(\gamma_{c}=1.6 \times 10^{5}, 3.3 \times 10^{5}\right)$, where a cooling break in the EED is expected, strongly suggests that the second break in the EED derived from the modeling is indeed the cooling break.

The observed spectral shape in both the low and high energy SED components are reproduced in our homogenous model by a change of electron index $\Delta p=p_{3}-p_{2}=2.0$. Such a large break in the EED is in contrast to the canonical cooling break $\Delta p=p_{3}-p_{2}=1.0$ that produces a spectral index change of $\Delta \alpha=0.5$, as predicted for homogenous models (e.g., Longair 1994). An attempt to model the data fixing $\Delta p=p_{3}-p_{2}=1.0$ gave unsatisfactory results, and hence this is not an option; a large spectral break is needed. It would be tempting to speculate that what we observe is not a cooling break, but rather something that results from a characteristic of the acceleration process which is not understood and that, therefore, does not bind us to the $\Delta p=1.0$ constraint. But we would then have to attribute to shear fortuity the fact that the Lorentz factors where this break 
takes place are very close to the Lorentz factors where cooling is actually expected.

The question that naturally arises is why, although the EED break postulated by the homogeneous model is at nearly the same energy as the expected cooling break, the spectral break observed is stronger. Such strong breaks are the rule rather than the exception in some non-thermal sources like pulsarwind nebulae and extragalactic jets (see Reynolds 2009) and the explanations that have been given relax the assumption of a homogenous emitting zone, invoking gradients in the physical quantities describing the system (Marscher 1980). In all inhomogeneous models, electrons are injected at an inlet and are advected downstream, suffering radiative losses that result in the effective size of the source declining with increasing frequency for a given spectral component. In sources where the beaming of the emitted radiation is the same throughout the source (this is the case for non-relativistic flows or for relativistic flows with small velocity gradients), the spectral break formed is stronger than the canonical $\Delta \alpha=0.5$ if the physical conditions change in such a way that the emissivity at a given frequency increases downstream (Wilson 1975; Coleman \& Bicknell 1988; Reynolds 2009).

If, in addition to these considerations, we allow for significant relativistic velocity gradients, either in the form of a decelerating flow (Georganopoulos et al. 2003) or the form of a fast spine and slow sheath flow (Ghisellini et al. 2005), the resulting differential beaming of the emitted radiation can result in spectral breaks stronger that $\Delta \alpha \approx 0.5$. Studies of the SEDs of sources with different jet orientations (e.g., radio galaxies and blazars) can help to understand the importance of differential beaming, and therefore of relativistic velocity gradients in these flows. Because in all these models the volume of the source emitting at a given frequency is connected to the predicted spectral break, it should be possible to use the variability timescale at different frequencies to constrain the physics of the inhomogeneous flow.

\subsection{Physical Properties of Mrk 421}

As mentioned in Section 5.3, the SED emerging from the multifrequency campaign is the most complete and accurate representation of the low/quiescent state of Mrk 421 to date. This data provided us with an unprecedented opportunity to constrain and tune state-of-the-art modeling codes. In Section 6 we modeled the SED within two different frameworks: a leptonic and a hadronic scenario. Both models are able to represent the overall SED. As can be seen in Figures 9 and 11, the leptonic model fits describe the observational data somewhat better than the hadronic model fits; yet we also note here that, in this paper, the leptonic model has one more free parameter than the hadronic model. A very efficient way of discriminating between the two scenarios would be through multiwavelength variability observations. It is, however, interesting to discuss the differences between the two model descriptions we presented above.

\subsubsection{Size and Location of the Emitting Region}

The characteristic size to which the size of the emitting region must be compared is the gravitational radius of the Mrk 421 black hole. For a black hole mass of $\sim 2-9 \times 10^{8} M_{\odot}$ (Barth et al. 2003; Wu et al. 2002), the corresponding size is $R_{g} \approx$ $0.5-2.0 \times 10^{14} \mathrm{~cm}$. In the hadronic model the source size can be as small as $R=4 \times 10^{14} \mathrm{~cm}$ (larger source sizes cannot be ruled out though; see Section 6.1), within one order of magnitude of the gravitational radius. The consequence is a dense synchrotron photon energy density that facilitates frequent interactions with relativistic protons, resulting in a strong reprocessed/cascade component which leads to a softening of the spectrum occurring mostly below $100 \mathrm{MeV}$. The Fermi-LAT analysis presented in this paper (which used the instrument response function given by P6_V3_DIFFUSE) is not sensitive to these low energies and hence the evaluation of this potential softening in the spectrum will have to be done with future analyses (and more data). This will potentially allow the accurate determination of spectra down to photon energies of $\sim 20 \mathrm{MeV}$ with LAT.

The leptonic model can accommodate a large range of values for $R$, as long as it is not so compact that internal $\gamma \gamma$ attenuation becomes too strong and absorbs the $\mathrm{TeV} \gamma$-rays. In the particular case of $t_{\mathrm{var}}=1$ day, which is supported by the low activity and low multifrequency variability observed during the campaign, $R=5 \times 10^{16} \mathrm{~cm}$, that is two to three orders of magnitude larger than the gravitational radius. Under the assumption that the emission comes from the entire (or a large fraction of the) cross-section of the jet, and assuming a conical jet, the location of the emitting region would be given by $L \sim R / \theta$, where $\theta \sim 1 / \Gamma \sim 1 / \delta$. Therefore, under these assumptions, which are valid for large distances $\left(L \gg R_{g}\right.$ ) when the outflow is fully formed, the leptonic model would put the emission region at $L \sim 10^{3}-10^{4} R_{g}$. We note, however, that since the $R$ for the leptonic model is considered an upper limit on the blob size scale (see Equation (1)), this distance should be considered as an upper limit as well.

\subsubsection{Particle Content and Particle Acceleration}

The particle contents predicted by the hadronic and leptonic scenarios are different by construction. In the hadronic scenario presented in Section 6.1, the dominantly radiating particles are protons, secondary electron/positron pairs, muons, and pions, in addition to the primary electrons. In the leptonic scenario, the dominantly radiating particles are the primary electrons only. In both cases, the distribution of particles is clearly non-thermal and acceleration mechanisms are required.

In the leptonic scenario, the PL index $p_{1}=2.2$, which is the canonical particle spectral index from efficient first-order Fermi acceleration at the fronts of relativistic shocks, suggests that this process is at work in Mrk 421. For electrons to be picked up by first-order Fermi acceleration in perpendicular shocks, their Larmor radius is required to be significantly larger than the width of the shock, which for electron-proton plasmas is set by the Larmor radius of the dynamically dominant particles (electrons or protons). The large $\gamma_{\min }\left(=8 \times 10^{2}\right)$ provided by the model implies that electrons are efficiently accelerated by the Fermi mechanism only above this energy and that below this energy they are accelerated by a different mechanism that produces an extremely hard electron distribution. Such pre-acceleration mechanisms have been discussed in the past (e.g., Hoshino et al. 1992). The suggestion that the Fermi mechanism picks up only after $\gamma_{\min }\left(=8 \times 10^{2}\right)$ suggests a large thickness of the shock, which would imply that the shock is dominated by (cold) protons. We refer the reader to the Fermi-LAT paper on Mrk 501 (Abdo et al. 2011b) for more detailed discussion on this topic. In addition, in Sections 6.2 and 7.1 we argued that the second break $\gamma_{\text {brk, } 2} m_{e} c^{2}(\sim 200 \mathrm{GeV})$ is probably due to synchrotron cooling (the electrons radiate most of their energy before exiting the region of size $R)$, but the first break $\gamma_{\text {brk, } 1} m_{e} c^{2}(\sim 25 \mathrm{GeV})$ must be related to the acceleration mechanism; and hence the 
leptonic model also requires that electrons above the first break are accelerated less efficiently. At this point it is interesting to note that the one-zone SSC model of Mrk 501 in 2009 (where the source was also observed mostly in a quiescent state), returned $\gamma_{\text {brk, } 1} m_{e} c^{2} \sim 20 \mathrm{GeV}$ with essentially the same spectral change (0.5) in the electron distribution (Abdo et al. 2011b). Therefore, the first break (presumably related to the acceleration mechanism) is of the same magnitude and located approximately at the same energy for both Mrk 421 and Mrk 501, which might suggest a common property in the quiescent state of HSP BL Lac objects detected at TeV energies.

The presence of intrinsic high energy breaks in the EED electron energy distribution has been observed in several of the Fermi-LAT blazars (see Abdo et al. 2009, 2010a). As reported in Abdo et al. (2010a), this characteristic was observed on several FSRQs, and it is present in some low-synchrotron-peaked BL Lac objects, and a small number of intermediate-synchrotronpeaked BL Lac objects; yet it is absent in all 1LAC HSP BL Lac objects. In this paper (as well as in Abdo et al. 2011b), we claim that such a feature is also present in HSP BL Lac objects like Mrk 421 and Mrk 501, yet for those objects, the breaks in the EED can only be accessed through proper SED modeling because they are smaller in magnitude, and somewhat smoothed in the high energy component. We note that, for HSP BL Lac objects, the high energy bump is believed to be produced by the EED upscattering seed photons from a wide energy range (the synchrotron photons emitted by the EED itself) and hence all the features from the EED are smoothed out. On the other hand, in the other blazar objects like FSRQs, the high energy bump is believed to be produced by the EED upscattering (external) seed photons which have a "relatively narrow" energy range. In this latter case (external Compton), the features of the EED may be directly seen in the gamma-ray spectrum. Another interesting observation is that, at least for one of the FSRQs, 3C 454.3, the location and the magnitude of the break seems to be insensitive to flux variations (Ackermann et al. 2010). If the break observed in Mrk 421 and Mrk 501 is of the same nature as that of 3C 454.3, we should also expect to see this break at the same location $(\sim 20 \mathrm{GeV})$ regardless of the activity level of these sources.

In the hadronic scenario of Figure 9, the blazar emission comes from a compact ( $R \sim$ a few $R_{g}$ ) highly magnetized emission region, which should be sufficiently far away from the central engine so that the photon density from the accretion disk is much smaller than the density of synchrotron photons. The gyroradius of the highest energy protons $\left(R_{L}=\gamma_{p, \max } m_{p} c^{2} /(e B)\right.$ in Gaussian-cgs units) is $\sim 1.4 \times 10^{14} \mathrm{~cm}$, which is a factor of about three times smaller than the radius of the spherical region responsible for the blazar emission $\left(R=4 \times 10^{14} \mathrm{~cm}\right)$, hence (barely) fulfilling the Hillas criterium. The small size of the emitting region, the ultra-high particle energies and the somewhat higher (by factor of about five) particle energy density with respect to the magnetic energy density imply that this scenario requires extreme acceleration and confinement conditions.

\subsubsection{Energetics of the Jet}

The power of the various components of the flow differs in the two models. In the SPB model, the particle energy density is about a factor of $\sim 5$ higher than the magnetic field energy density and the proton energy density dominates over that of the electrons by a factor of $\sim 40$. In the leptonic model, the electron energy density dominates over that of the magnetic field by a factor of 10. By construction, the leptonic model does not constrain the proton content and hence we need to make assumptions about the number of protons. It is reasonable to use charge neutrality to justify a comparable number of electrons and protons. Under this assumption, the leptonic model predicts that the energy carried by the electrons (which is dominated by the parameter $\gamma_{\min } \sim 10^{3}$ ) is comparable to that carried by the (cold) protons.

The overall jet power determined by the hadronic model is $P_{\text {jet }}=4.4 \times 10^{44} \mathrm{erg} \mathrm{s}^{-1}$. For the day variability timescale leptonic model, assuming one cold proton per radiating electron, the power carried by the protons would be $4.4 \times 10^{43} \mathrm{erg} \mathrm{s}^{-1}$, giving a total jet power of $P_{\text {jet }}=1.9 \times 10^{44} \mathrm{erg} \mathrm{s}^{-1}$. In both cases, the computed jet power is a small fraction $\left(\sim 10^{-2}\right.$ to $\left.10^{-3}\right)$ of the Eddington luminosity for the supermassive black hole in Mrk $421\left(2 \times 10^{8} M_{\odot}\right)$, which is $L_{\text {Edd }} \sim 10^{46}-10^{47} \mathrm{erg} \mathrm{s}^{-1}$.

\subsection{Interpretation of the Reported Variability}

In Section 3 we reported the $\gamma$-ray flux/spectral variations of Mrk 421 as measured by the Fermi-LAT instrument during the first 1.5 years of operation. The flux and spectral index were determined on seven-day-long time intervals. We showed that, while the $\gamma$-ray flux above $0.3 \mathrm{GeV}$ flux changed by a factor of about three, the PL photon index variations are consistent with statistical fluctuations (Figure 1) and the spectral variability could only be detected when comparing the variability in the $\gamma$-ray flux above $2 \mathrm{GeV}$ with the one from the $\gamma$-ray flux below $2 \mathrm{GeV}$. It is worth pointing out that, in the case of the TeV blazar Mrk 501, the $\gamma$-ray flux above $2 \mathrm{GeV}$ was also found to vary more than the $\gamma$-ray flux below $2 \mathrm{GeV}$. Yet unlike Mrk 421, Mrk 501 was less bright at $\gamma$-rays and the flux variations above $2 \mathrm{GeV}$ seem to be larger, which produced statistically significant changes in the photon index from the PL fit in the energy range $0.3-400 \mathrm{GeV}$ (see Abdo et al. 2011b). In any case, it is interesting to note that in these two (classical) TeV objects, the flux variations above a few $\mathrm{GeV}$ are larger than the ones below a few $\mathrm{GeV}$, which might suggest that this is a common property in HSP BL Lac objects detected at TeV energies.

In Section 3 we also showed (see Figures 2-4) that the X-ray variability is significantly higher than that in the $\gamma$-ray band measured by Fermi-LAT. In addition, we also saw that the $15-50 \mathrm{keV}$ (BAT) and the $2-10 \mathrm{keV}$ (ASM) fluxes are positively correlated, and that the BAT flux is more variable than the ASM flux. In other words, when the source flares in X-rays, the X-ray spectrum becomes harder.

In order to understand this long baseline $\mathrm{X}$-ray $/ \gamma$-ray variability within our leptonic scenario, we decomposed the $\gamma$ ray bump of the SED into the various contributions from the various segments of the EED, according to our one-zone SSC model, in a similar way as it was done in Tavecchio et al. (1998). This is depicted in Figure 13. The contributions of different segments of the EED are indicated by different colors. As shown, the low-energy electrons, $\gamma_{\min } \leqslant \gamma<\gamma_{b r, 1}$, which are emitting synchrotron photons up to the observed frequencies $\simeq 5.2 \times 10^{15} \mathrm{~Hz}$, dominate the production of $\gamma$-rays up to the observed photon energies of $\sim 20 \mathrm{GeV}$ (green line). The contribution of higher energy electrons with Lorentz factors $\gamma_{b r, 1} \leqslant \gamma<\gamma_{b r, 2}$ is pronounced within the observed synchrotron range $5 \times 10^{15}-10^{17} \mathrm{~Hz}$, and at $\gamma$-ray energies from $\sim 20 \mathrm{GeV}$ up to $\sim \mathrm{TeV}$ (blue line). Finally, the highest energy tail of the electron energy distribution, $\gamma \geqslant \gamma_{b r, 2}$, responsible for the production of the observed X-ray synchrotron continuum $(>0.5 \mathrm{keV})$ generates the bulk of $\gamma$-rays with the observed energies $>\mathrm{TeV}$ (purple line). Because of the electrons upscattering the broad energy range of synchrotron photons, the emission 

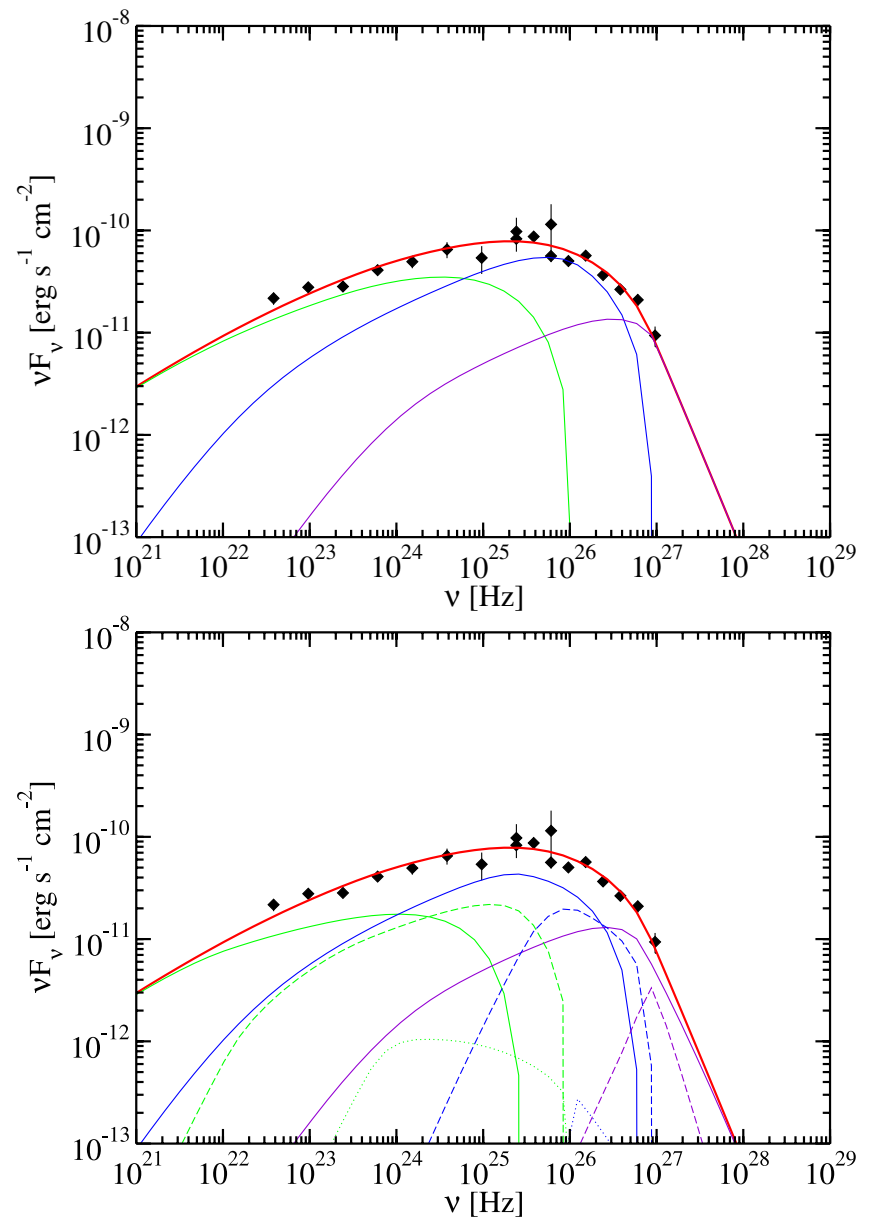

Figure 13. Decomposition of the high energy bump of the SSC continuum for Mrk 421. The data points are the same as in the high energy bump from Figure 11. The SSC fit to the average spectrum is denoted by the red solid curve. Top: contributions of the different segments of electrons Comptonizing the whole synchrotron continuum (green curve: $\gamma_{\min }<\gamma<\gamma_{b r, 1}$; blue curve: $\gamma_{b r, 1}<\gamma<\gamma_{b r, 2}$; purple curve: $\left.\gamma_{b r, 2}<\gamma\right)$. Bottom: contributions of the different segments of electrons (as in the top panel) Comptonizing different segments of the synchrotron continuum (solid curves: $v<v_{b r, 1} \simeq 5.3 \times 10^{15}$ $\mathrm{Hz}$; dashed curves: $v_{b r, 1}<v<v_{b r, 2} \simeq 1.3 \times 10^{17} \mathrm{~Hz}$; dotted curves, corresponding to $v>v_{b r, 2}$ ).

of the different electron segments are somewhat connected, as shown in the bottom plot of Figure 13. Specifically, the low energy electrons have also contributed to the TeV photon flux through the emitted synchrotron photons which are being upscattered by the high energy electrons. Hence, changes in the number of low energy electrons should also have an impact on the $\mathrm{TeV}$ photon flux. However, note that the synchrotron photons emitted by the high energy electrons, which are upscattered in the Klein-Nishina regime, do not have any significant contribution to the gamma-ray flux, thus changes in the number of high energy electrons (say $\gamma>\gamma_{b r, 2}$ ) will not significantly change the $\mathrm{MeV} / \mathrm{GeV}$ photon flux.

Within our one-zone SSC scenario, the $\gamma$-rays measured by Fermi-LAT are mostly produced by the low energy electrons $\left(\gamma \leqslant \gamma_{b r, 1}\right)$ while the X-rays seen by ASM and BAT are mostly produced by the highest energy electrons $\left(\gamma \geqslant \gamma_{b r, 2}\right)$. In this scenario, the significantly higher variability in the X-rays with respect to that of $\gamma$-rays suggests that the flux variations in Mrk 421 are dominated by changes in the number of the highest energy electrons. Note that the same trend is observed in the $\mathrm{X}$-rays (ASM versus BAT) and $\gamma$-rays (below versus above
$2 \mathrm{GeV}$ ); the variability in the emission increases with the energy of the radiating electrons.

The greater variability in the radiation produced by the highest energy electrons is not surprising. The cooling timescales of the electrons from synchrotron and inverse Compton (in the Thomson regime) losses scale as $t \propto \gamma^{-1}$, and hence it is expected that the emission from higher energy electrons will be the most variable. However, since the high energy electrons are the ones losing their energy fastest, in order to keep the source emitting in X-rays, injection (acceleration) of electrons up to the highest energies is needed. This injection (acceleration) of high energy electrons could well be the origin of the flux variations in Mrk 421. The details of this high energy electron injection could be parameterized by changes in the parameters $\gamma_{b r, 2}, p_{3}$, and $\gamma_{\max }$ within the framework of the one-zone SSC model that could result from episodic acceleration events (Perlman et al. 2005). The characterization of the SED evolution (and hence SSC parameter variations) will be one of the prime subjects of the forthcoming publications with the multiinstrument variability and correlation during the campaigns in $2009^{130}$ and 2010. ${ }^{131}$ We note here that SSC models, both onezone and multizone (e.g., Graff et al. 2008), predict a positive correlation between the X-rays and the $\mathrm{TeV} \gamma$-rays measured by IACTs. Indeed, during the 2010 campaign the source was detected in a flaring state with the TeV instruments (see ATel 2443). Such an X-ray/TeV correlation has been established in the past for this object (see Maraschi et al. 1999), although the relation is not simple. Sometimes it is linear and at other times it is quadratic (e.g., Fossati et al. 2008). The complexity of this correlation is also consistent with our one-zone SSC model; the X-rays are produced by electrons with $\gamma>\gamma_{b r, 2}$, while the $\mathrm{TeV}$ photons are produced by electrons with $\gamma>\gamma_{b r, 1}$, and is indirectly affected by the electrons with $\gamma<\gamma_{b r, 1}$ through the emitted synchrotron photons that are used as seed photons for the inverse Compton scattering (see the bottom plot of Figure 13).

We also note that the one-zone SSC scenario presented here predicts a direct correlation on the basis of simultaneous data sets between the low energy gamma-rays (from Fermi) and the SMA and optical frequencies, since both energy bands are produced by the lowest energy electrons in the source. On the other hand, our SPB model fit does not require such a strict correlation, but there could be a loose correlation if electrons and protons are accelerated together. In particular, a direct correlation with zero time lag between the millimeter radio frequencies and the $\gamma$-rays is not expected in our SPB model because the radiation at these two energy bands are produced at different sites. The radiation in the X-ray and $\gamma$-ray bands originates from the primary electrons, and from the protons and secondary particles created by proton-initiated processes, respectively. Consequently, although a loose correlation between the X-ray and $\gamma$-ray bands can be expected if protons and electrons are accelerated together, a strict correlation with zero time lag is rather unlikely in our model fit.

During the 2009 and 2010 campaigns, Mrk 421 was very densely sampled during a very long baseline (4.5 and 6 months for the 2009 and 2010 campaigns, respectively) and hence these data sets will provide excellent information for performing a

\footnotetext{
${ }^{130}$ For details of the 2009 campaign, see the URL https://confluence.slac.stanford.edu/display/GLAMCOG/Campaign +on+Mrk421+(Jan+2009+to+May+2009).

${ }^{131}$ For details of the 2010 campaign, see the URL

https://confluence.slac.stanford.edu/display/GLAMCOG/Campaign +on+Mrk421+\%28December+2009+to+December+2010\% 29.
} 
very detailed study of these multiband relations. In particular, during the campaign in 2010, there were regular observations with VLBA and SMA, which will allow us to study with a greater level of detail the relationship between the rising parts of the low energy and high energy bumps, where the predictions from the leptonic and hadronic models differ.

\section{CONCLUSIONS}

In this work, we reported on the $\gamma$-ray activity of Mrk 421 as measured by the LAT instrument on board the Fermi satellite during its first 1.5 years of operation, from 2008 August 5 (MJD 54683) to 2009 March 12 (MJD 55248). Because of the large leap in capabilities of LAT with respect to its predecessor, EGRET, this is the most extensive study of the $\gamma$-ray activity of this object at $\mathrm{GeV}$ photon energies to date. The Fermi-LAT spectrum (quantified with a single PL function) was evaluated for seven-day-long time intervals. The average photon flux above $0.3 \mathrm{GeV}$ was found to be $(7.23 \pm 0.16) \times 10^{-8} \mathrm{ph} \mathrm{cm}^{-2} \mathrm{~s}^{-1}$, and the average photon index $1.78 \pm 0.02$. The photon flux changed significantly (up to a factor of about three) while the spectral variations were mild. The variations in the PL photon index were not statistically significant, yet the light curves and variability quantification below and above $2 \mathrm{GeV}$ showed that the high $\gamma$-ray energies vary more than the low energy $\gamma$-rays. We found $F_{\text {var }}(E<$ $2 \mathrm{GeV})=0.16 \pm 0.04$ while $F_{\text {var }}(E>2 \mathrm{GeV})=0.33 \pm 0.04$. We compared the LAT $\gamma$-ray activity in these two energy ranges $(0.2-2 \mathrm{GeV}$ and $>2 \mathrm{GeV})$ with the $\mathrm{X}$-ray activity recorded by the all-sky instruments RXTE/ASM (2-10 keV) and Swift/ BAT $(15-50 \mathrm{keV})$. We found that X-rays are significantly more variable than $\gamma$-rays, with no significant $(\lesssim 2 \sigma)$ correlation between them. We also found that, within the X-ray and $\gamma$-ray energy bands, the variability increased with photon energy. The physical interpretation of this result within the context of the one-zone SSC model is that the variability in the radiation increases with the energy of the electrons that produce them, which is expected given the radiating timescales for synchrotron and inverse Compton emission.

We also presented the first results from the 4.5 month long multifrequency campaign on Mrk 421, which lasted from 2009 January 19 (MJD 54850) to 2009 June 1 (MJD 54983). During this time period, the source was systematically observed from radio to $\mathrm{TeV}$ energies. Because of the low activity and low variability shown during this campaign, the compiled data provided us with the best SED yet of Mrk 421 in the low/ quiescent state.

The broadband SED was modeled with two different scenarios: a leptonic (one-zone SSC) model and a hadronic model (SPB). Both frameworks are able to describe reasonably well the average SED, implying comparable powers for the jet emission, which constitute only a small fraction $\left(\sim 10^{-2}\right.$ to $\left.10^{-3}\right)$ of the Eddington luminosity. However, those models differ on the predicted environment for the blazar emission: the leptonic scenario constrains the size to be $R \lesssim 10^{4} R_{g}$, the magnetic field to $B \sim 0.05 \mathrm{G}$ and particles (electrons) with energies up to $\sim 5 \times 10^{13} \mathrm{eV}$ while, if $\alpha_{e}=\alpha_{p}$, our hadronic scenario implies a size of the emitting region of a few $R_{g}$, a magnetic field $B \sim 50$ $\mathrm{G}$ and particles (protons) with energies up to $\sim 2 \times 10^{18} \mathrm{eV}$, which requires extreme conditions for particle acceleration and confinement.

The leptonic scenario suggests that the acceleration of the radiating particles (electrons) is through diffusive shock acceleration in relativistic shocks mediated by cold protons, and that this mechanism accelerates particles (electrons) less efficiently above an energy of $\sim 25 \mathrm{GeV}$, which is comparable to what was reported in Abdo et al. (2011b) for another classical TeV blazar, Mrk 501. In addition, unlike what was observed for Mrk 501, in the case of Mrk 421 a stronger-than-canonical electron cooling break was required to reproduce the observed SED, which might suggest that the blazar emitting region is inhomogeneous.

Within the SSC model (Figure 11), the observed X-ray/ $\gamma$-ray variability during the first 1.5 years of Fermi operation indicates that the flux variations in Mrk 421 are produced by acceleration of the highest energy electrons, which radiate in the $\mathrm{X}$-ray and $\mathrm{TeV}$ bands, and lose energy, radiating as they do so in the optical and $\mathrm{GeV}$ range. In our hadronic model (Figure 9), a rather loose correlation between the X-ray and $\gamma$-ray bands is expected if electrons and protons are accelerated together. A forthcoming publication will report on whether these emission models can reproduce the multiband flux variations observed during the intensive campaigns on Mrk 421 performed in 2009 and 2010. Those studies should help us distinguish between the hadronic and the leptonic scenarios and eventually lead to a better understanding of one of the fundamental mysteries of blazars: how flux variations are produced.

The authors of the paper thank the anonymous referee for very well-organized and constructive comments that helped improve the quality and clarity of this publication.

The Fermi-LAT Collaboration acknowledges the generous support of a number of agencies and institutes that have supported the Fermi-LAT Collaboration. These include the National Aeronautics and Space Administration and the Department of Energy in the United States, the Commissariat à l'Energie Atomique and the Centre National de la Recherche Scientifique/ Institut National de Physique Nucléaire et de Physique des Particules in France, the Agenzia Spaziale Italiana and the Istituto Nazionale di Fisica Nucleare in Italy, the Ministry of Education, Culture, Sports, Science and Technology (MEXT), High Energy Accelerator Research Organization (KEK), and Japan Aerospace Exploration Agency (JAXA) in Japan, and the K. A. Wallenberg Foundation, the Swedish Research Council and the Swedish National Space Board in Sweden. Additional support for science analysis during the operations phase is gratefully acknowledged from the Istituto Nazionale di Astrofisica in Italy and the Centre National d'Études Spatiales in France.

The MAGIC Collaboration thanks the Instituto de Astrofísica de Canarias for the excellent working conditions at the Observatorio del Roque de los Muchachos in La Palma. The support of the German BMBF and MPG, the Italian INFN, the Swiss National Fund SNF, and the Spanish MICINN is gratefully acknowledged. This work was also supported by the Marie Curie program, by the CPAN CSD2007-00042 and MultiDark CSD2009-00064 projects of the Spanish Consolider-Ingenio 2010 programme, by grant DO02-353 of the Bulgarian NSF, by grant 127740 of the Academy of Finland, by the YIP of the Helmholtz Gemeinschaft, by the DFG Cluster of Excellence "Origin and Structure of the Universe," and by the Polish MNiSzW Grant N N203 390834.

We acknowledge the use of public data from the Swift and RXTE data archives. The Metsähovi team acknowledges the support from the Academy of Finland for the observing projects (numbers 212656, 210338, among others). This research has made use of data obtained from the National Radio Astronomy Observatory's Very Long Baseline Array (VLBA), projects BK150, BP143, and BL149 (MOJAVE). The National 
Radio Astronomy Observatory is a facility of the National Science Foundation operated under cooperative agreement by Associated Universities, Inc. The St. Petersburg University team acknowledges support from the Russian RFBR foundation via grant 09-02-00092. AZT-24 observations are made within an agreement between Pulkovo, Rome and Teramo observatories. This research is partly based on observations with the $100 \mathrm{~m}$ telescope of the MPIfR (Max-Planck-Institut für Radioastronomie) at Effelsberg, as well as with the Medicina and Noto telescopes operated by INAF-Istituto di Radioastronomia. RATAN-600 observations were supported in part by the RFBR grant 08-02-00545 and the OVRO $40 \mathrm{~m}$ program was funded in part by NASA (NNX08AW31G) and the NSF (AST-0808050). The Submillimeter Array is a joint project between the Smithsonian Astrophysical Observatory and the Academia Sinica Institute of Astronomy and Astrophysics and is funded by the Smithsonian Institution and the Academia Sinica. M. Villata organized the optical-to-radio observations by GASP-WEBT as the president of the collaboration. The Abastumani Observatory team acknowledges financial support by the Georgian National Science Foundation through grant GNSF/ST07/4-180.

\section{REFERENCES}

Abdo, A. A., et al. 2009, ApJ, 699, 817

Abdo, A. A., et al. 2010a, ApJ, 710, 1271

Abdo, A. A., et al. 2010b, ApJS, 188, 405

Abdo, A. A., et al. 2010c, ApJ, 716, 30

Abdo, A. A., et al. 2011a, Science, 331, 739

Abdo, A. A., et al. 2011b, ApJ, 727, 129

Acciari, V. A., et al. 2009, ApJ, 703, 169

Acciari, V. A., et al. 2011, ApJ, in press (arXiv:1106.1210)

Ackermann, M., et al. 2010, ApJ, 721, 1383

Aharonian, F., et al. 2002, A\&A, 393, 89

Aharonian, F., et al. 2003, A\&A, 410, 813

Aharonian, F., et al. 2005, A\&A, 437, 95

Ajello, M., et al. 2008, ApJ, 673, 96

Ajello, M., et al. 2009a, ApJ, 690, 367

Ajello, M., et al. 2009b, ApJ, 699, 603

Albert, J., et al. 2007a, ApJ, 663, 125

Albert, J., et al. 2007b, ApJ, 669, 862

Albert, J., et al. 2007c, Nucl. Instrum. Methods, A583, 494

Albert, J., et al. 2008, ApJ, 674, 1037

Aleksić, J., et al. 2010, A\&A, 519, A32

Aliu, E., et al. 2009, Astropart. Phys., 30, 293

Aller, H. D., Aller, M. F., Latimer, G. E., \& Hodge, P. E. 1985, ApJS, 59, 513

Angelakis, E., Fuhrmann, L., Marchili, N., Krichbaum, T. P., \& Zensus, J. A. 2008, Mem. Soc. Astron. Ital., 79, 1042

Atwood, W. B., et al. 2009, ApJ, 697, 1071

Barth, A. J., Ho, L. C., \& Sargent, W. L. W. 2003, ApJ, 583, 134

Barthelmy, S. D., et al. 2005, Space Sci. Rev., 120, 143

Błażejowski, M., et al. 2005, ApJ, 630, 130

Bradt, H. V., Rothschild, R. E., \& Swank, J. H. 1993, A\&AS, 97, 355

Burrows, D. N., et al. 2005, Space Sci. Rev., 120, 165

Cao, X. 2003, ApJ, 599, 147

Coleman, C. S., \& Bicknell, G. V. 1988, MNRAS, 230, 497

Daum, A., et al. 1997, Astropart. Phys., 8, 1

Donnarumma, I., et al. 2009, ApJ, 691, L13

Edelson, R. A., \& Krolik, J. H. 1988, ApJ, 333, 646

Edelson, R., et al. 2002, ApJ, 568, 610

Finke, J. D., Dermer, C. D., \& Böttcher, M. 2008, ApJ, 686, 181

Finke, J. D., Razzaque, S., \& Dermer, C. D. 2010, ApJ, 712, 238

Fitzpatrick, E. L. 1999, PASP, 111, 63

Fossati, G., et al. 2008, ApJ, 677, 906

Franceschini, A., et al. 2008, A\&A, 487, 837

Fuhrmann, L., et al. 2008, A\&A, 490, 1019

Fukugita, M., Shimasaku, K., \& Ichikawa, T. 1995, PASP, 107, 945

Gaidos, J. A., et al. 1996, Nature, 383, 319
Gehrels, N., et al. 2004, ApJ, 611, 1005

Georganopoulos, M., \& Kazanas, D. 2003, ApJ, 594, L27

Ghisellini, G., Tavvechio, F., \& Chiaberge, M. 2005, A\&A, 432, 401

Giannios, D., Uzdensky, D. A., \& Begelman, M. C. 2009, MNRAS, 395, L29

Giebels, B., Dubus, G., \& Khélifi, B. 2007, A\&A, 462, 29

Gilmore, R. C., Madau, P., Primack, J. R., Somerville, R. S., \& Haardt, F. 2009, MNRAS, 399, 1694

Graff, P. B., Georganopoulos, M., Perlman, E. S., \& Kazanas, D. 2008, ApJ, 689,68

Hartman, R. C., et al. 1999, ApJS, 123, 79

Horan, D., et al. 2009, ApJ, 695, 596

Hoshino, M., Arons, J., Gallant, Y. A., \& Langdon, A. B. 1992, ApJ, 390, 454

Kalberla, P. M. W., et al. 2005, A\&A, 440, 775

Katarzyński, K., Lenain, J.-P., Zech, A., Boisson, C., \& Sol, H. 2008, MNRAS, 390, 371

Katarzyński, K., Sol, H., \& Kus, A. 2003, A\&A, 410, 101

Kneiske, T. M., Bretz, T., Mannheim, K., \& Hartmann, D. H. 2004, A\&A, 413, 807

Komissarov, S. S., \& Falle, S. A. E. G. 1997, MNRAS, 288, 833

Kovalev, Y. Y., Nizhelsky, N. A., Kovalev, Y. A., Berlin, A. B., Zhekanis, G. V., Mingaliev, M. G., \& Bogdantsov, A. V. 1999, A\&AS, 139, 545

Kovalev, Y. Y., et al. 2005, AJ, 130, 2473

Krawczynski, H., et al. 2001, ApJ, 559, 187

Krennrich, F., et al. 2002, ApJ, 575, L9

Lister, M. L., et al. 2009, AJ, 137, 3718

Lobanov, A. P. 2005, arXiv:astro-ph/0503225

Longair, M. S. 1994, High Energy Astrophysics (Cambridge: Cambridge Univ. Press)

Maraschi, L., et al. 1999, ApJ, 526, L81

Marscher, A. P. 1980, ApJ, 235, 386

Marscher, A. P. 1983, ApJ, 264, 296

Marscher, A. P., et al. 2008, Nature, 452, 966

Massaro, E., Perri, M., Giommi, P., \& Nesci, R. 2004a, A\&A, 413, 489

Massaro, E., Perri, M., Giommi, P., Nesci, R., \& Verrecchia, F. 2004b, A\&A, 422, 103

Mattox, J. R., et al. 1996, ApJ, 461, 396243

Mücke, A., \& Protheroe, R. J. 2001, Astropart. Phys., 15, 121

Mücke, A., Protheroe, R. J., Engel, R., Rachen, J. P., \& Stanev, T. 2003, Astropart. Phys., 18, 593

Nandra, K., et al. 1997, ApJ, 476, 70

Nilsson, K., et al. 2007, A\&A, 475, 199

Paneque, D. 2009, in ASP Conf. Ser. 427, Accretion and Ejection in AGNs: A Global View, ed. L. Maraschi et al. (San Francisco, CA: ASP), 277

Perlman, E. S., et al. 2005, ApJ, 625, 727

Pichel, A. (VERITAS Collaboration) 2009, Proc. 31st ICRC (Łódz̀) arXiv:0908.0010

Poole, T. S., et al. 2008, MNRAS, 383, 627

Punch, M., et al. 1992, Nature, 358, 477

Rebillot, P. F., et al. 2006, ApJ, 641, 740

Reynolds, S. P. 2009, ApJ, 703, 662

Richards, J., et al. 2011, ApJS, 194, 29

Roming, P. W. A., et al. 2005, Space Sci. Rev., 120, 95

Schlegel, D. J., Finkbeiner, D. P., \& Davis, M. 1998, ApJ, 500, 525

Sikora, M., Madejski, G., Moderski, R., \& Poutanen, J. 1997, ApJ, 484, 108

Sokolovsky, K. V., Kovalev, Y. Y., Lobanov, A. P., Savolainen, T., Pushkarev, A. B., \& Kadler, M. 2010, arXiv:1001.2591

Stecker, F. W., Malkan, M. A., \& Scully, S. T. 2006, ApJ, 648, 774

Tavani, M., et al. 2011, Science, 331, 736

Tavecchio, F., Maraschi, L., \& Ghisellini, G. 1998, ApJ, 509, 608

Teräsranta, H., et al. 1998, A\&AS, 132, 305

Tramacere, A., Giommi, P., Perri, M., Verrecchia, F., \& Tosti, G. 2009, A\&A, 501,879

Vaughan, S., et al. 2003, MNRAS, 345, 1271

Venturi, T., et al. 2001, A\&A, 379, 755

Villata, M., Raiteri, C. M., Lanteri, L., Sobrito, G., \& Cavallone, M. 1998, A\&AS, 130, 305

Villata, M., et al. 2008, A\&A, 481, L79

Villata, M., et al. 2009, A\&A, 504, L9

Wagner, R. M. 2008, MNRAS, 385, 119

Wilson, A. S. 1975, A\&A, 43, 1

Wilson-Hodge, C. A., et al. 2011, ApJ, 727, L40

Wu, X.-B., Liu, F. K., \& Zhang, T. Z. 2002, A\&A, 389, 742 